\title{
THE
}

\section{Planarity and out-of-plane vibrational modes of tryptophan and tyrosine in biomolecular modeling}

\author{
Faramarz Joodaki \\ University of Rhode Island \\ Lenore M. Martin \\ University of Rhode Island, martin@uri.edu \\ Michael L. Greenfield \\ University of Rhode Island, greenfield@uri.edu
}

Follow this and additional works at: https://digitalcommons.uri.edu/cmb_facpubs

The University of Rhode Island Faculty have made this article openly available.
Please let us know how Open Access to this research benefits you.

This is a pre-publication author manuscript of the final, published article.

Terms of Use

This article is made available under the terms and conditions applicable towards Open Access Policy Articles, as set forth in our Terms of Use.

\section{Citation/Publisher Attribution}

Joodaki, F., Martin, L. M., \& Greenfield, M. L. (2019). Planarity and out-of-plane vibrational modes of tryptophan and tyrosine in biomolecular modeling. Phys. Chem. Chem. Phys., 21, 23943-23965. doi: 10.1039/C9CP04798K

Available at: http://dx.doi.org/10.1039/C9CP04798K 


\title{
Journal Name
}

\section{ARTICLE TYPE}

Cite this: DOI: $10.1039 / x x x x x x x x x x$

Accepted Date

DOI: 10.1039/xxxxxxxxxx

www.rsc.org/journalname

\section{Planarity and out-of-plane vibrational modes of trypto- phan and tyrosine in biomolecular modeling $\dagger$}

\author{
Faramarz Joodaki, ${ }^{a}$ Lenore M. Martin, ${ }^{b}$ and Michael L. Greenfield ${ }^{* a}$
}

Tryptophan and tyrosine are aromatic amino acids that play significant roles in the folding processes of proteins at water-membrane interfaces because of their amphipathic structures. Employing appropriate heteroaromatic molecular structures are essential for obtaining accurate dynamics and predictive capabilities in molecular simulations of these amino acids. In this study, molecular dynamics simulations that applied the most recent version of the CHARMM36 force field were conducted on aqueous solutions of tryptophan and of tyrosine. Geometric analysis and dynamics quantified how aromatic rings deviated from planar structures and exhibited out-ofplane fluctuations. Radial distribution functions showed possible biological significance because extent of ring planarity slightly affected local water concentrations near aromatic rings. Instantaneous all-atom normal mode analysis (NMA) and Fourier transformation of time autocorrelation functions of out-of-plane displacements were applied to study out-of-plane vibrations of atoms in those rings. NMA started with minimum energy configurations and then averaged over fluctuations in aqueous solution. The frequencies and frequency patterns that were obtained for tryptophan and tyrosine with CHARMM36 differed from literature reports of Raman spectra, infrared spectra, and frequencies calculated using quantum mechanics, with some out-of-plane modes found at higher frequencies. Effects of imposing improper torsion potentials and changing torsion angle force constants were investigated for all atoms in the rings of tryptophan and tyrosine. Results show that these coarse force fields variations only affect planarity and out-of-plane vibrations of atoms within the rings, not other vibrations. Although increasing improper torsion force constants reduced deviations from aromatic ring planarity significantly, it increased out-of-plane mode frequencies. Reducing torsion angle force constants (with and without improper torsions) shifted modes to lower frequencies. A combination of decreasing most torsion angle force constants for ring atoms in both amino acids and including improper torsion forces attained frequencies and frequency patterns for out-of-plane normal modes that were more similar to literature spectra. These force field variations decreased the extents of out-of-plane vibrations within the heteroaromatic rings of tryptophan, especially around the nitrogen atom in the ring, but not within the heteroaromatic ring of tyrosine. Conclusions were unaffected by peptide endgroup, water, or simulation ensemble.

\section{Introduction}

Molecular simulation of biomolecules at the atomic scale owes its success to reliable empirical force fields (FFs). For 40 years,

\footnotetext{
${ }^{a}$ Department of Chemical Engineering, University of Rhode Island, Kingston, Rhode Island 02881, United States.

${ }^{b}$ Department of Cell and Molecular Biology, University of Rhode Island, Kingston, Rhode Island 02881, United States.

$\dagger$ Electronic Supplementary Information (ESI) available: [details of any supplementary information available should be included here]. See DOI: 10.1039/cXCP00000x/
}

various sets of FF parameters have been developed to achieve more accurate and realistic structures of biological systems,, $1+3$ and simulations have successfully described biological phenomena. ${ }^{3}$ CHARMM is one of the most successful and advantageous FFs, and it is widely used in biomolecular simulations.24/5 During the past three decades, CHARMM parameters have been extended to improve representation of biological compounds such as amino acids, nucleic acids, lipids, and carbohydrates. $\underline{1316}[11$

The predictive ability of molecular simulation is strongly based on the choice of FF terms and accuracy of FF parameters. $\frac{5.12}{12}$ Several simulation studies demonstrated that employing different 
FFs in protein and peptide folding simulations leads to different folded structures and free energy surfaces. $\frac{5|15| 16}{16}$ Since the structure of a folded protein links to its functionality, $\frac{17}{17}$ this diversity of obtained folded structures suggests different protein functionalities. Even when employing the same FF set with different parameter versions (for example CHARMM22 and CHARMM22/CMAP), simulation results were altered. 16 Some studies pointed out a bias effect in modern FFs, including CHARMM and AMBER, on the shape of folded proteins. 18 For instance, a biased tendency for folded proteins to adopt a helical shape has been reported in molecular dynamics (MD) simulations 14]18. Hence, implementing appropriate FF parameters plays a crucial role in predicting biomolecular structures and atom motions in a system. ${ }^{1 / 13}$ It is essential to improve understanding of how parameter changes affect modeling results. ${ }^{19} \mathrm{FF}$ parameter improvements continue to evolve and enhance the agreement between simulation and experimental observables. 13

A variety of studies have demonstrated significant roles for two heteroaromatic amino acids, tryptophan (Trp, W) and tyrosine (Tyr, $\mathrm{Y}$ ), in the folding processes of proteins at watermembrane interfaces. These amino acids have special aromatictype side chains with non-uniform, amphipathic structures that anchor a folded protein at the water-membrane interface. 20,23 For instance, Trp has a vital role in maintaining the structure and function of gramicidin channels at the surface of a cell membrane. ${ }^{22}$ Some studies showed that a Trp residue in an antimicrobial peptide forms a hydrogen bond with the phosphate head group of membrane lipids 24 and leads to deeper peptide insertion into membrane bilayers. $25 \mid 26$ The side chain of Trp, a rigid indole ring, has the greatest hydrophobic surface area among all aromatic amino acids. 22

The FF parameters of Trp and Tyr, shown as zwitterions in Fig. 1, have been a focus for improvement 2728 because of their unusual heteroaromatic structures. The high participation of the indole ring in protein folding has motivated considering $\pi / \pi$ and $\mathrm{CH} / \pi$ interactions of Trp in the improvement of FF (CHARMM22) parameters. 27 A related example is the modification of cation/ $\pi$ interactions in the FF parameters that reflect interactions between the phenolic ring of Tyr and choline-containing phospholipid head groups in order to match observed physical properties of this interaction to quantum calculation results. $\frac{28}{28}$ Competition between cation $/ \pi$ and salt bridge interactions as sources of stabilizing forces have also been identified. ${ }^{29}$ All of these examples illustrate the importance of using accurate structures for Trp and Tyr for the assembly of simulated biological systems. Assigning appropriate FF parameters for these two amino acids plays a significant role in improving the overall reliability of peptide simulation results.

Modeling heteroaromatic rings has long been controversial because their symmetry 30 and chemical reactivity 31 suggest that their geometry may differ from that of typical aromatic rings. According to molecular orbital theory and by using the linear combination of atomic orbitals approximation, the lowest energy level for an aromatic ring, such as a benzene molecule, has a symmetric and planar wavefunction. $\frac{32}{32}$ Experimental and quantum calculations also provide evidence for the planarity of heteroaromatic rings. For instance, X-ray crystallography of N-acetyl-tryptophan-

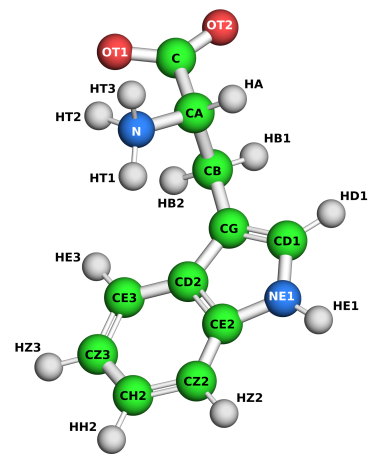

(a)

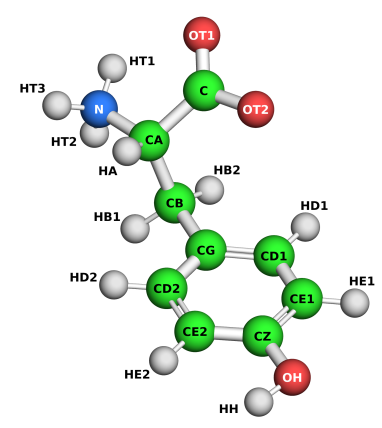

(b)
Fig. 1 Zwitterionic forms of (a) tryptophan and (b) tyrosine. Labels show atom ID assignments used in molecular simulations.

methylamide,, $33 \sqrt{34}$ microwave spectroscopy of indole, 35 and $a b$ initio calculations of indole, $\sqrt[36 \mid 37]{3 \text { 3-methylindole, }}, \frac{38}{38}$ and D-Trp 39 confirmed that all atoms in the indole ring of Trp are in the same plane for minimum-energy conformers. X-ray crystallography 40 and CASSCF quantum mechanics calculations ${ }^{41}$ have indicated a planar structure for $\mathrm{p}$-cresol, which is identical to the aromatic side chain of Tyr. For substituted heteroaromatic compounds that arise in asphaltene chemistry, Li and Greenfield demonstrated via quantum mechanics calculations that phenol- and pyrrole-based multi-ring planar architectures have lower total potential energies compared to possible nonplanar structures. 42

Ring planarity is not static; Raman and infrared spectra of Trp and Tyr indicate out-of-plane vibrational modes of atoms in side-chain aromatic rings. 3943 Sh 4 Slight deviations of 0.1 to $0.4^{\circ}$ were found for indole ring torsion angles for a Trp zwitterion in quantum mechanics calculations that implicitly included solvent effects. $\frac{46}{}$ Car-Parrinello molecular dynamics simulations 50 of an isolated benzene molecule demonstrated that an average planar structure is a consequence of symmetric out-of-plane fluctuations. Most rings were not instantaneously planar: C-C-C-C torsion angles often fluctuated up to $10^{\circ}$ and rarely up to $20^{\circ}$. An ultrahigh-resolution crystallography study of $\alpha$-lytic protease has shown that interactions between phenylalanine (Phe228) and threonine (Thr181) side chains cause out-of-plane distortions of the Phe aromatic ring, 51 showing that average out-of-plane deviations are possible in certain circumstances. An intent of the present work is to explore the extent that rings of Trp and Tyr fluctuate from planarity under a classical force field.

If an atom in an aromatic ring and the three adjacent atoms bonded to it are all found to be in the same plane, then that portion of the ring has a planar structure and the sum of angles between bonds around the central atom should be $360^{\circ}$. If the sum is less than $360^{\circ}$, then these four atoms cannot be in the same plane. We describe an aromatic ring structure as "closer to planar" if the average sum of angles around ring atoms over time is closer to $360^{\circ}$. Therefore, we compute one measure of planarity by averaging the sum of bond angles around each atom in the ring. A second measure quantifies the perpendicular distance 
from a central atom to the plane of its three connected neighbors.

The CHARMM FF typically applies an out-of-plane bending term to force four specific atoms into a plane. ${ }^{2 / 3}$ This term is a quadratic improper torsion (dihedral) function that contributes to maintaining the planarity and chirality characteristics of a central atom. ${ }^{252}$ Implementing this term is also critical for dynamics and vibrational analysis in order to achieve a proper force field near the minimum energy geometry. ${ }^{52}$ In the CHARMM22 FF, quadratic Urey-Bradley and improper torsion potential functions contribute to in-plane and out-of-plane deformation vibrations of atoms, respectively. MacKerell et al. $\frac{53}{53}$ report that they implemented these functions as a final optimization step for vibrational spectra only if there was a discrepancy between calculated results on the basis of CHARMM parameters and available data. In other words, Urey-Bradley and improper torsion parameters were considered to optimize the fits of FF parameters to vibrational spectra from ab initio calculations or from Raman and infrared spectroscopies.653 MacKerell et al. demonstrated that considering improper torsion angle forces for the peptide bond (in CHARMM22) led to vibrational analysis results for two out-of-plane modes of an $\mathrm{N}$-methylacetamide molecule that were in good agreement with quantum calculations and experimental results. 53

In the recent version of CHARMM FF (CHARMM36, C36), improper torsion potentials are incorporated for atoms in the heteroaromatic rings of histidine ${ }^{7-[9}$ and nucleic acid bases, $\frac{10[11}{11}$ yet they do not arise for atoms in the aromatic rings of tryptophan, tyrosine, or phenylalanine. It seems that C36 relies instead on torsion potentials in their rings to achieve average planar structures and out-of-plane vibrations at appropriate frequencies. In an aromatic ring with a potentially planar structure, a small change in torsion angles around atoms in the rings can cause nonplanarity. This approach, considering only torsion angles in the FF equation for the aromatic rings of Trp and Tyr, coupled with the significant roles played by the structure of these two amino acids in biological phenomena and a prevalence for nonplanar aromatic rings in molecular simulations of peptides, encouraged us to investigate the planarity of these rings during molecular simulations.

Some frequencies in Raman and infrared spectra correspond to out-of-plane vibrations of atoms in the aromatic rings of Trp and Tyr that have been described as out-of-plane bending, torsional internal coordinate, ring deformation, and ring twisting. 43.46 49 Improper torsion angles and changes to torsion angle force constants for these atoms could alter out-of-plane vibrational frequencies. Hence, we were motivated to calculate the effect of force fields on out-of-plane ring vibrations.

In this work, we report the effects of applying different FF sets on the structure and dynamics of atoms in the aromatic rings of Trp and Tyr in MD simulations. The C36 FF has been employed as a default FF. We then added an improper torsion angle potential for atoms in the rings of Trp and Tyr. Different values of improper torsion force constants have been evaluated. In some cases, we changed the torsion angle force constant around the aromatic rings of Trp and Tyr to investigate its effects on aromatic ring planarity. First, a geometric analysis is applied to the MD results to quantify how changes in each FF set affect the maintenance of planar structures. Next, we applied all-atom normal mode analysis (NMA) to results of equilibrated MD simulations to investigate how changing FF parameters affected out-of-plane vibrations of atoms in the rings of Trp and Tyr. This work helps us to study the effects of improper and regular torsion angle parameters on the planarity and out-of-plane vibrations of atoms in the rings of Trp and Tyr, which are important because the amplitudes of these motions directly impact interactions of heteroaromatic rings with neighborhood molecules. Results are compared to Raman and infrared spectra and to quantum mechanics calculations results available in the literature.43/44/46/49 Results with zwitterions are compared to those of Trp and Tyr with methylamide terminal groups.

\section{METHODOLOGY}

\section{Force Field Setup}

The recent version of CHARMM36 (C36) ${ }^{7-9} \mathrm{FF}$ was used for all MD simulations and minimizations. We investigated planarity of heteroaromatic ring geometries by adding an improper torsion angle potential for the atoms in the rings of tryptophan (Trp, W) and tyrosine (Tyr, Y) within molecular simulations.

Table 1 lists the sets of improper torsion angles for atoms that we have considered in the rings of Trp ( 9 sets) and Tyr (6 sets). In each set, the improper torsion angle $\varphi$ for the four atoms is defined as the angle between the plane going through the first three atoms in the set and a second plane going through the last three atoms. We implemented the improper torsion potential as

$$
U_{\text {improper }}=K_{\text {imp }}\left(\varphi-\varphi_{0}\right)^{2}
$$

where $K_{\mathrm{imp}}$ is the improper torsion angle force constant and $\varphi_{0}$ is the equilibrium improper torsion angle. C36 FF uses eq 1 for other systems, ${ }^{53}$ such as histidine. Table 2 lists the different $K_{\text {imp }}$ that were applied for different sets of minimizations and MD simulations in this work. The applied $K_{\mathrm{imp}}$ are of the same order of magnitude as those used in C36 for similar systems. According to $\mathrm{X}$-ray crystallography and $a b$ initio calculation results, $\underline{33|34| 38}$ all atoms of heteroaromatic rings fluctuate about a plane at equilibrium; thus we set $\varphi_{0}=0$ for all improper torsion angle sets.

Next, we investigated the effects of varying torsion angle force constants on the planarity of the Trp and Tyr rings. The torsion angle sets that C36 applies for atoms in the rings of Trp and Tyr are shown in Table $\mathrm{S} 1$, ESI $\uparrow .7$ Any change in a torsion angle for atoms in an aromatic ring deforms planarity, so these terms interact with the improper torsion angle terms. The torsion angle potential equation used in $\mathrm{C} 36 \mathrm{FF}$ has the form 53

$$
U_{\text {torsion }}=K_{\text {tor }}(1+\cos (n \chi-\delta))
$$

where $K_{\text {tor }}$ is the side chain torsion angle force constant, $\chi$ and $\delta$ are the torsion angle and an equilibrium torsion angle parameter, and the integer $n$ affects the form of the potential. $n$ equals 2 and $\delta$ equals $\pi$ for all torsion angles with parameters that are varied in this work.

For most torsion angle sets listed in Table S1, ESI $\nmid$ (40 sets for Trp and 20 sets for Tyr), we decreased $K_{\text {tor }}$ by either 0.5 , 1 , or $1.5 \mathrm{kcal} / \mathrm{mol}$, as listed in Table 2 Since $K_{\text {tor }}$ of torsion angle sets 
Table 1 Improper torsion angle sets considered for Trp and Tyr during this work.

\begin{tabular}{cl|cl}
\hline \multicolumn{2}{c|}{$\begin{array}{c}\text { Trp } \\
\text { Set }\end{array}$} & Atom Names & Set \\
\hline 1 & CG CD2 CD1 CB & 1 & CG CD2 CD1 CB \\
2 & CD1 CG NE1 HD1 & 2 & CD1 CG CE1 HD1 \\
3 & NE1 CD1 CE2 HE1 & 3 & CE1 CD1 CZ HE1 \\
4 & CE2 NE1 CD2 CZ2 & 4 & CZ CE1 CE2 OH \\
5 & CZ2 CE2 CH2 HZ2 & 5 & CE2 CZ CD2 HE2 \\
6 & CH2 CZ2 CZ3 HH2 & 6 & CD2 CE2 CG HD2 \\
7 & CZ3 CH2 CE3 HZ3 & & \\
8 & CE3 CZ3 CD2 HE3 & & \\
9 & CD2 CE2 CG CE3 & &
\end{tabular}

${ }^{a}$ Atom names are taken from Fig. 1. These names differ from the parameter names used in a C36 parameter file.

Table 2 FF sets used in energy minimizations and MD.

\begin{tabular}{|c|c|c|c|c|}
\hline $\begin{array}{c}\text { Amino } \\
\text { acid }\end{array}$ & FF Set & FF set label & $\begin{array}{c}K_{\text {imp }}{ }^{a} \\
\left(\mathrm{kcal} / \mathrm{mol}-\mathrm{rad}^{2}\right)\end{array}$ & $\begin{array}{c}K_{\text {tor }}-K_{\text {tor }}^{C 36 b} \\
(\mathrm{kcal} / \mathrm{mol})\end{array}$ \\
\hline \multirow{12}{*}{ Trp } & $0^{c}$ & 0,0 & 0 & 0 \\
\hline & 1 & 5,0 & 5 & 0 \\
\hline & 2 & 10,0 & 10 & 0 \\
\hline & 3 & 20,0 & 20 & 0 \\
\hline & 4 & 90,0 & 90 & 0 \\
\hline & 5 & $0,-0.5$ & 0 & -0.5 \\
\hline & 6 & $10,-0.5$ & 10 & -0.5 \\
\hline & 7 & $20,-0.5$ & 20 & -0.5 \\
\hline & 8 & $0,-1$ & 0 & -1 \\
\hline & 9 & $10,-1$ & 10 & -1 \\
\hline & 10 & $15,-1$ & 15 & -1 \\
\hline & 11 & $20,-1$ & 20 & -1 \\
\hline \multirow{11}{*}{ Tyr } & $\overline{0^{c}}$ & 0,0 & 0 & 0 \\
\hline & 1 & 5,0 & 5 & 0 \\
\hline & 2 & 10,0 & 10 & 0 \\
\hline & 3 & 20,0 & 20 & 0 \\
\hline & 4 & 90,0 & 90 & 0 \\
\hline & 5 & $0,-1$ & 0 & -1 \\
\hline & 6 & $10,-1$ & 10 & -1 \\
\hline & 7 & $20,-1$ & 20 & -1 \\
\hline & 8 & $0,-1.5$ & 0 & -1.5 \\
\hline & 9 & $10,-1.5$ & 10 & -1.5 \\
\hline & 10 & $20,-1.5$ & 20 & -1.5 \\
\hline
\end{tabular}

${ }^{a} K_{\mathrm{imp}}$ is the improper torsion force constant that we added to the C36 FF for sets of atoms in heteroaromatic rings (sets in Table 1)

${ }^{b} K_{\text {tor }}$ is the torsion angle force constant that we changed for atoms in the rings. $K_{\text {tor }}^{C 36}$ is each C36 FF torsion force constant (Table S1, ESI $\dagger$ ).

${ }^{c}$ C36 default parameters

37-40 of Trp are small, we did not change them.

Both $K_{\text {imp }}$ and $K_{\text {tor }}$ control bond forces that restore planarity of a ring vibrating around its nominally planar equilibrium structure. Different combinations of $K_{\mathrm{imp}}$ and $K_{\text {tor }}$ provide various extents of planar geometries and realistic dynamics for the aromatic rings of Tyr and Trp. The first set used for both molecules was the C36 FF in which $K_{\text {imp }}=0$ and $K_{\text {tor }}$ were unchanged.

\section{Simulations}

The cationic ammonium (protonated amino) group and anionic carboxylate group found in zwitterionic amino acids were implemented for most simulations of Tyr and Trp molecules (Fig. 1). $p K_{a}$ is low for the carboxyl group and high for the amino group in aqueous solution; $[56$ hence, these two amino acids exist mostly as zwitterions at neutral or physiologic $\mathrm{pH} . \underline{45[56}$ To check if charged end groups affect out-of-plane motions of ring atoms, MD simulations using C36 were repeated for Trp and Tyr with methylamide terminal groups (Fig. S1. ESI $\dagger$ ).

For energy minimization, systems that contain a single molecule of either Trp or Tyr in a cubic vacuum box with a dimension of $40 \times 40 \times 40 \AA^{3}$ were prepared. The size of each system was big enough so a Trp or Tyr molecule could not interact with itself via periodic boundary conditions. The conjugate gradient method was used for energy minimization. These minimized structures were used for calculating reference modes.

For MD simulations, one molecule of zwitterionic Trp or Tyr was solvated in the box by 1439 or 1144 water molecules, respectively. The TIP3P model was used for the water molecules. 57 First, the systems of Trp-water and Tyr-water were minimized. Following minimization, NPT MD simulation was performed on each system with a time step of $1 \mathrm{fs}$ until energy, temperature, and volume fluctuations showed equilibration (approximately $50 \mathrm{ps}$ ). MD simulation was continued for 250 ps after equilibrium, and pdb structural files were stored for every time step of this period. Such frequent output was required in order to monitor ring geometry due to its high vibrational frequencies. The last $200 \mathrm{ps}$ were used in our analysis. This method was repeated for all the different FF sets for both Trp and Tyr (Table 2).

All minimizations and simulations were performed using the NAMD ${ }^{58}$ package. Three-dimensional periodic boundary conditions were applied. A cutoff of $12 \AA$, switch distance of $10 \AA$, and pair list distance of $14 \AA$ were set in all simulations. Neighbor lists were rebuilt every 10 steps. Particle mesh Ewald ${ }^{59}$ was used for long range electrostatic interactions. Langevin dynamics with a damping coefficient of $1 \mathrm{ps}^{-1}$ were applied to maintain a temperature of 298K, while Nose-Hoover Langevin piston pressure control was used with barostat oscillation and damping time scales of $0.2 \mathrm{ps}$ and $0.1 \mathrm{ps}$ to provide a constant 1 atm pressure. $60 \mid 61$

To confirm that results were independent of temperature and pressure control algorithms, NVE MD simulations using C36 were performed starting from a configuration that was as close as possible to the average volume found in the NPT simulations.

\section{Geometric Analysis}

To investigate the planarity of aromatic rings, two methods were applied for each snapshot along an equilibrated MD trajectory. Carbon atoms with $\mathrm{sp}^{2}$ hybridizations in a ring are bonded as shown in Fig. 2 for central atom $i$ connected to atoms $j, k$, and $l$.

In the first method, the sum of bond angles centered around each atom except hydrogen in the ring was calculated (for instance, $\alpha+\beta+\gamma$ for atom $i$ in Fig. 2a), and the average of this sum was obtained over 200 ps during the MD simulation. In a planar structure, the sum of bond angles around each atom in the 


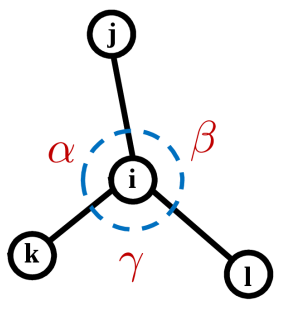

(a)

Fig. 2 Atom $i$ has bonds with atoms $j, k$, and $l$. Planarity is quantified using (a) the sum of the angles $(\alpha+\beta+\gamma)$ around atom $i$ and (b) the distance $d_{\perp}$ from atom $i$ to the plane containing atoms $j, k$, and $l$.

ring is $360^{\circ}$. If the sum is less than $360^{\circ}$, atoms will form a nonplanar structure. This sum can never be greater than $360^{\circ}$. By this analysis, we can determine how an applied FF set affects the average sum of angles and "dimpling" from a plane.

In the second method, the distance $\left(d_{\perp}\right)$ of the central atom from the plane containing three connected atoms was calculated (Fig. 23). Atoms $i, j, k$, and $l$ are located in the same plane when $d_{\perp}$ is zero. $d_{\perp}$ can be negative or positive depending on the position of the central atom $(i)$ on each side of the plane $(j-k-l) . d_{\perp}$ was calculated for all atoms in the rings of Trp and Tyr, and averages and standard deviations were calculated over 200 ps. The standard deviation of $d_{\perp}$ contributes to understanding the magnitude of the out-of-plane fluctuations. This was quantified further by a time autocorrelation function $C(\tau)$, averaged over all time origins $t$ within the trajectory,

$$
C(\tau)=\frac{\left\langle d_{\perp}(t) d_{\perp}(t+\tau)\right\rangle-\left\langle d_{\perp}\right\rangle^{2}}{\left\langle d_{\perp}{ }^{2}\right\rangle-\left\langle d_{\perp}\right\rangle^{2}}
$$

where time difference $\tau$ is an integer multiple of the time step $\delta t$.

Fourier transformation (FT) has been applied to the autocorrelation function results to convert from the time domain into the frequency domain so out-of-plane fluctuation frequencies of atoms in the aromatic rings can be investigated. These frequencies clearly demonstrate how employing different FF sets affects out-of-plane fluctuations of the atoms in the aromatic rings.

\section{All-Atom Normal Mode Analysis}

All-atom normal mode analysis (NMA) was implemented to investigate further the vibrational motions of atoms in the heteroaromatic rings of Trp and Tyr within a classical force field. NMA provides all vibrational frequencies in a system. ${ }^{54 / 55}$ Movements of atoms in molecules are linear combinations of these normal modes. In this method, the potential energy, which is written on a basis of atomic coordinates (X), is expanded about a local minimum or stationary point $\left(\mathrm{X}_{0}\right)$ by the Taylor series $\frac{54|55| 62}{6}$

$U(\mathbf{X})=U\left(\mathbf{X}_{0}\right)+U^{\prime}\left(\mathbf{X}_{0}\right)\left(\mathbf{X}-\mathbf{X}_{0}\right)+\frac{1}{2}\left(\mathbf{X}-\mathbf{X}_{0}\right)^{T} U^{\prime \prime}\left(\mathbf{X}_{0}\right)\left(\mathbf{X}-\mathbf{X}_{0}\right)+\ldots$

Because the first derivative of potential energy $U^{\prime}\left(\mathbf{X}_{0}\right)$ is zero at stationary points, the second term on the right-hand side drops out. $U^{\prime \prime}\left(\mathbf{X}_{0}\right)$ is the second derivative of potential energy with re- spect to Cartesian coordinates of each atom, which is called the Hessian matrix $\boldsymbol{H}$. Rearranging equation 4 leads to

$$
U(\mathbf{X})=U\left(\mathbf{X}_{0}\right)+\frac{1}{2}\left(\mathbf{X}-\mathbf{X}_{0}\right)^{T} \mathbf{Z}^{T} \mathbf{Z} \boldsymbol{H} \boldsymbol{Z}^{T} \mathbf{Z}\left(\mathbf{X}-\mathbf{X}_{0}\right)+\ldots
$$

where $\boldsymbol{Z}$ is a matrix that ensures $\mathbf{Z} \mathbf{H} \mathbf{Z}^{T}$ is a diagonal matrix, and the product $\boldsymbol{Z}^{T} \boldsymbol{Z}$ equals the identity matrix. If there are $N$ atoms, then the dimensions of the Hessian matrix are designated as $3 N \times$ $3 N$. Taylor series terms of higher than second order are neglected in the harmonic approximation.

Calculating the Hessian matrix leads to the normal modes of each molecule. First, the Hessian matrix needs to be converted to an equivalent force-constant matrix $\underline{54[62}$

$$
\boldsymbol{F}=\boldsymbol{M}^{-1 / 2} \boldsymbol{H} \boldsymbol{M}^{-1 / 2}
$$

where $\boldsymbol{M}$ is a diagonal matrix containing the atomic masses. The eigenvalues and eigenvectors of matrix $\boldsymbol{F}$ are calculated by solving

$$
F V_{i}=\lambda_{i} V_{i}
$$

where $\lambda_{i}$ is an eigenvalue and $V_{i}$ is an orthonormal eigenvector that has a dimension of $3 N \times 1$. Similarly, Lagrangian mechanics applied to the kinetic and potential energy leads to $N$ linear differential equations. By assuming an oscillatory solution for these equations, we also reach the same eigenvalue-eigenvector problem (eq7). Hence, each $\lambda_{i}$ can be used to calculate the frequency $\left(v_{i}\right)$ of normal mode $i$ by $\underline{54 \mid 55}$

$$
v_{i}=\sqrt{\lambda}_{i} / 2 \pi
$$

Each obtained frequency $\left(v_{i}\right)$ corresponds to a different mode of motion, and each eigenvector (the normal mode vector, $V_{i}$ ) specifies the vibrational motions of atoms that correspond to that frequency, i.e. each normal mode vector indicates the direction that all atoms move in that mode. Six modes with eigenvalues of zero within numerical precision represent the translation and rotation of these molecules; hence, there are $3 N-6$ total vibrational modes in a system of $N$ atoms. ${ }^{54[55}$ Vibrational modes are calculated here without regard to selection rules that dictate which modes show infrared or Raman activity.

We developed a package for NMA using the $\mathrm{C}++$ language. In this package, the Hessian matrix was calculated analytically using the Cartesian coordinates of atoms and the FF parameters. The chain rule was applied to calculate the second derivative of potential energy with respect to Cartesian coordinates. The C++ LAPACK library 63 has been used for numerically solving eigenvalue-eigenvector problems. Furthermore, this package provides an animation of each vibrational mode that may be viewed using $\mathrm{VMD} \sqrt{64}$ software.

To build the Hessian matrix, potential energy expressions for bond, angle, torsion, improper torsion, Urey-Bradley, and nonbonded (Lennard-Jones and Coulomb) interactions were used to calculate the total potential energy. Periodic boundary conditions were applied. The same cutoff and switch functions that we have used for MD simulations were applied for Lennard-Jones and short range Coulomb interactions. We applied the same po- 
tential energy equations that are defined in NAMD and C36 FFs, except for long range electrostatic interactions. The electrostatic potential equation has the form 53

$$
U_{e l}=\sum_{i} \sum_{j} \frac{q_{i} q_{j}}{4 \pi \varepsilon_{0} r_{i j}}
$$

where $q_{i}$ and $q_{j}$ are the partial atomic charges of atoms $i$ and $j$, respectively, $\varepsilon_{0}$ is the permittivity of free space, and $r_{i j}$ is the distance between atoms $i$ and $j$. The second derivative of the electrostatic potential for atoms with a large $r_{i j}$ is close to zero. Therefore, we chose to omit long range electrostatic interactions in NMA calculations. We have verified the implementation of each analytical derivative that leads to the Hessian matrix by comparing to numerical derivatives for small molecules.

For NMA calculation, only FF parameters and molecule coordinates are needed to calculate vibrational modes $V_{i}$. We applied NMA on the energy minimization results for each FF set (12 sets for Trp and 11 sets for Tyr) to find all possible vibrational modes. We divided all normal vibrational modes into three categories: out-of-plane, planar, and backbone/linker modes. In out-of-plane modes, atom vibrations deformed the planarity of the ring. In planar modes, ring atoms moved within the ring plane. Backbone/linker modes involved atoms not in the rings. Our main interest in this work is out-of-plane modes. These were identified by visualizing animations of all normal modes using VMD. 64 Monitoring $d_{\perp}$ for each mode led to a consistent choice (ESI $\dagger$ ).

\section{Comparing Normal Modes across Simulations}

For each FF set, out-of-plane normal modes obtained from the minimized structures of isolated amino acids were considered as reference modes. Applying different FFs can cause similar modes to arise at different frequencies. To find similar modes, a technique using the dot product of two modes from different FF sets as a guide was applied. We start with an eigenvector $V_{i}^{A}$ for mode $i$ from FF set A $(0,0)$ and calculate its dot products with all the modes $j$ from another FF set "B". The largest dot product indicates the mode $V_{j}^{B}$ in the second FF that is most similar to mode $V_{i}^{A}$ in FF $(0,0)$. Our package systematically finds similar modes on the basis of this method.

To perform averaging within each defined FF set, MD simulations of Trp and Tyr in solution were conducted. NMA was applied for each time step ( $\delta t=1 \mathrm{fs}$ ) along an equilibrated MD trajectory (200 ps). Since all of the derivatives were calculated analytically, building the Hessian matrix in our package is fast even for larger systems; more time is spent calculating eigenvalues and eigenvectors because the speed of this calculation decreases with a scaling of $\sim N^{3}$ as the number of atoms in the system increases. By applying instantaneous NMA on a whole system of amino acid + water, a large number of modes is obtained. Most of these modes correspond to water molecule vibrations that are not related to the Trp or Tyr molecule of interest. Thus, instead of applying instantaneous NMA on the whole system for each trajectory, we applied it on just the Trp or Tyr molecule. To validate this assumption, using the C36 FF set as an example, we applied NMA on the entire system including water in a minimum energy configuration.

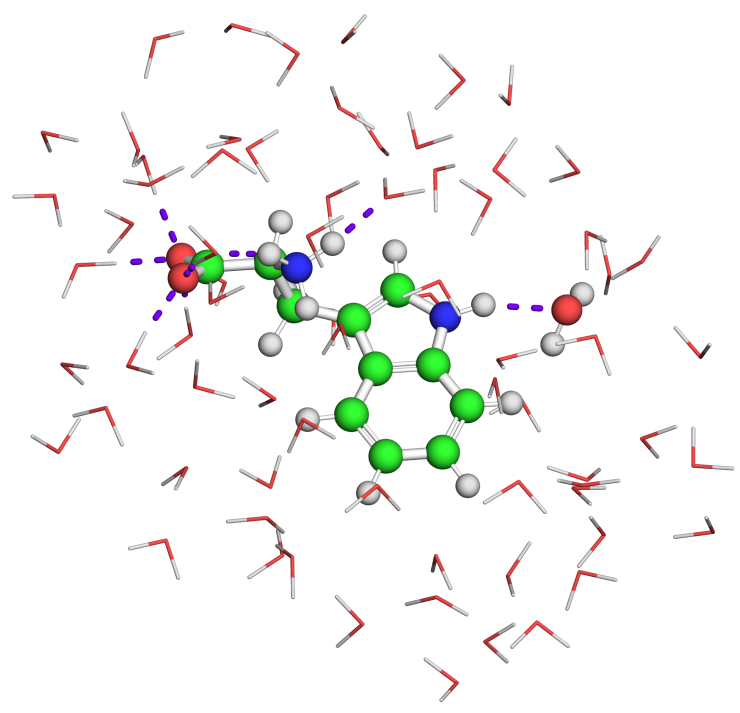

Fig. 3 Subset of the overall Trp+water system that was considered for NMA calculations. The purple dashed lines show hydrogen bonds between Trp molecule and water molecules that were identified using PyMOL. 65 The maximum distance for ideal hydrogen bonds and the maximum angle for a marginal hydrogen bond are $3.6 \AA$ and $63^{\circ}$, respectively.

To simplify the system, only water molecules whose atoms are closer than $6 \AA$ to any of the atoms of the Trp and Tyr molecules were included in the NMA calculation (see Fig. 3). NMA results for this system were compared to those from the system of zwitterion alone in a vacuum.

The next step was finding similar normal modes over 200 ps trajectories by using reference modes. First, dot products of reference eigenvectors and eigenvectors of the current snapshot were calculated. Since the positions and orientations of Trp and Tyr molecules changed during an MD simulation, the amino acid was translated and rotated to align with the reference structure (minimized structure) before calculating the dot product. Consequently, the eigenvectors of each configuration along a trajectory were also reoriented. The most similar modes have the greatest dot product that corresponds to the amino acid portion of the eigenvectors. Finally, an arithmetic average of wavenumbers for similar modes was calculated over 200 ps for each set of MD simulations, i.e. for each FF. This time is long enough to average over many vibrations within the local potential energy minimum. This method helps in understanding the effects of changing FF parameters on the resulting geometry (planarity) and out-of-plane normal modes of Trp and Tyr.

The instantaneous NMA method was also applied on systems of Trp and Tyr with methylamide terminal groups in water and on zwitterions sampled in the NVE ensemble (results in ESI $\dagger$ ).

\section{Results and Discussion}

\section{Geometric Analysis}

We performed MD simulations that employed different FF sets (Table 2) on aqueous solutions of either tryptophan (Trp, W) or tyrosine (Tyr, Y). We monitored the dynamic structures of these 
zwitterionic amino acids by applying geometric analysis on the results of equilibrated MD simulations. The same procedure employing C36 has been used for Trp and Tyr with methylamide terminal groups; results are provided in ESI $\dagger$, Figs. S2 and S3. As an example, results for the nitrogen atom (NE1) in the indole ring of Trp and gamma carbon (CG) in Tyr are plotted in Fig. 4 and Fig.5, respectively. Fig. 4 a shows the sum of the bond angles around NE1 as a function of simulation time when applying the C36 FF (Table 2 set 0 ). The sum around NE1 has significant differences from $360^{\circ}$ during the 200 ps simulated, indicating that the aromatic ring of Trp experiences a nonplanar structure around NE1 when using the C36 FF. Occasionally, the bond angle sum around NE1 reached as low as $330^{\circ}$. Fig. 4 p shows the results of monitoring the bond angle sum around NE1 when applying parameter set 4 (Table 2. $(90,0)$ ). In this FF set, an improper torsion potential with a force constant of $90 \mathrm{kcal} / \mathrm{mol}^{-\mathrm{rad}^{2}}$ has been adopted for atoms in the ring of Trp. As shown in Fig. 4 p, although there were some moments when the sum reached a minimum of $354.06^{\circ}$, the sum around NE1 was consistently closer to $360^{\circ}$ when such a stiff improper torsion potential was included. The sum around CG in Tyr reached a minimum around $353^{\circ}$ during MD simulations with the C36 FF (Fig. 5a). However, when applying an improper torsion function $\left(K_{\mathrm{imp}}=90 \mathrm{kcal} / \mathrm{mol}_{-} \mathrm{rad}^{2}\right)$ for all atoms in the ring of Tyr, the sum is closer to $360^{\circ}$. In both cases, since the sums were shifted closer to $360^{\circ}$, we can state that the structures of Trp around NE1 and Tyr around CG remained closer to planar when including improper torsion terms for these two heteroaromatic rings.

The deformation distance $\left(d_{\perp}\right)$ of atom NE1 from the base plane containing adjacent atoms HE1, CD1, and CE2 (Fig. 1) also quantifies the planarity of the heteroaromatic ring. Figs. 4 . and $4 \mathrm{~d}$ present plots of $d_{\perp}$ fluctuations calculated for NE1 by applying C36 and then by including the improper torsion $\left(K_{\mathrm{imp}}=\right.$ $90 \mathrm{kcal} / \mathrm{mol}^{-\mathrm{rad}^{2}}$ ) function, respectively. When including an energy term for improper torsion angles, the average of $d_{\perp}$ shifted closer to zero. In addition, the maximum deviation of $d_{\perp}$ decreased from $\pm 0.402 \AA$ to $\pm 0.163 \AA$ while its standard deviation decreased from \pm 0.108 to $\pm 0.043 \AA$ when applying an improper torsion function. Therefore we conclude that inclusion of improper torsion terms decreases out-of-plane displacement of NE1 and leads to Trp maintaining a more planar structure around NE1. Furthermore, geometric analysis results for CG demonstrate that adding an improper torsion term $\left(K_{\mathrm{imp}}=90 \mathrm{kcal} / \mathrm{mol}-\mathrm{rad}^{2}\right)$ to the C36 FF decreases the fluctuations in $d_{\perp}$ for the heteroaromatic ring in Tyr; maximum deviation of $d_{\perp}$ decreased from $\pm 0.216 \AA$ to $\pm 0.158 \AA$ and standard deviation decreased from $\pm 0.051 \AA$ to $\pm 0.032 \AA$. Including improper torsion angles had similar effects on the geometry results for other atoms in the heteroaromatic rings. Out-of-plane fluctuations for Trp and Tyr with methylamide terminal groups were comparable to those in zwitterions. Results are shown in Figs. S6 and S7, ESI $\dagger$. Comparable results also were obtained for NE1 of Trp and CG of Tyr in NVE MD simulations. (Figs. S10 and S11. ESI $\dagger$ )

To compare the effects of different FF modifications on the geometries of Trp and Tyr, the average sum of bond angles and the average out-of-plane deformation $d_{\perp}$ were calculated using
200 ps MD simulations of zwitterion in water. In the upper panel of Fig. 6, we plot the effects of different FF sets on the average sum of bond angles around each atom in the ring of Trp. NE1 has the highest difference from the average sum of $360^{\circ}$ in all FF sets. The FF set with an improper force constant of $90 \mathrm{kcal} / \mathrm{mol}^{-\mathrm{rad}^{2}}$ resulted in the most planar structure for the indole ring compared to other FF sets. Even the average sum of NE1 was $359.6^{\circ}$ in this case, which means NE1 stayed close to the plane of the indole ring throughout the MD simulation. By considering improper torsion and increasing $K_{\mathrm{imp}}$, the average sum around all atoms, not only $\mathrm{NE} 1$, was shifted closer to $360^{\circ}$, indicating that the indole ring of Trp maintained a planar structure. The lower panel of Fig. 6 shows the average $d_{\perp}$ of all atoms in the indole ring for each set of MD simulations using different FF parameters. Although in all sets the average $d_{\perp}$ was close to zero, the averages were closer to zero in the FF set with $K_{\text {imp }}=90 \mathrm{kcal} / \mathrm{mol}_{-} \mathrm{rad}^{2}$. These results indicate that out-of-plane displacement among adjacent atoms decreased and atoms formed a more planar structure as the improper torsion force constant increased. The lower panel of Fig. 6 also shows the standard deviation of each $d_{\perp}$, which demonstrates the extent of out-of-plane displacements. The standard deviation of $d_{\perp}$ decreased for all of the atoms in the heteroaromatic ring as $K_{\mathrm{imp}}$ was increased; this limited atom movements and caused them to fluctuate closer to the plane of the indole ring in Trp simulations.

The right side of Fig. 6 shows how making coarse changes to the coefficients for regular torsion angles around the ring affects the geometry results. In the FF sets in which the torsion angle force constants $\left(K_{\text {tor }}\right)$ of all atoms in the ring were decreased by $1 \mathrm{kcal} / \mathrm{mol}$, the spreads in the average $d_{\perp}$ of atoms from the ring were slightly larger than for set $(0,0)$. The average bond angle sums show a little less planarity when compared to those from simulations using the C36 FF. This result is plausible because decreasing all $K_{\text {tor }}$ decreases the energy penalty for out-of-plane displacements of atoms, and consequently the atom deviations from a planar ring structure increased.

Employing both modifications simultaneously leads to some cancellations. Out-of-plane fluctuations are smaller yet not as small as when torsion parameters were unchanged. Decreasing the $K_{\text {tor }}$ while including a term that accounts for improper torsion motions increased overall planarity.

The results of monitoring the planarity of atoms in Tyr simulated under different FF sets are compared in Fig. 7 For the C36 FF (label 0,0), there were differences in the average bond angle sum from $360^{\circ}$ for atoms in the ring of Tyr. These differences, which were smaller than those calculated for Trp, were reduced by incorporating an improper torsion potential term with higher $K_{\text {imp }}$. The strongest improper torsion potential $\left(K_{\mathrm{imp}}=90\right)$ contributed to a decrease in the standard deviation of $d_{\perp}$ for each atom in the ring, which indicates that the atoms fluctuated within a smaller amplitude perpendicular to the plane of Tyr. Gradually decreasing all of the original $K_{\text {tor }}$ caused the atoms of the Tyr ring to be generally less planar compared to the structure obtained using the C36 FF. Applying improper torsion on this decreased $K_{\text {tor }}$ FF set, for example $(10,-1)$, contributed toward the aromatic ring becoming more planar; however, the Tyr ring in this case was no 

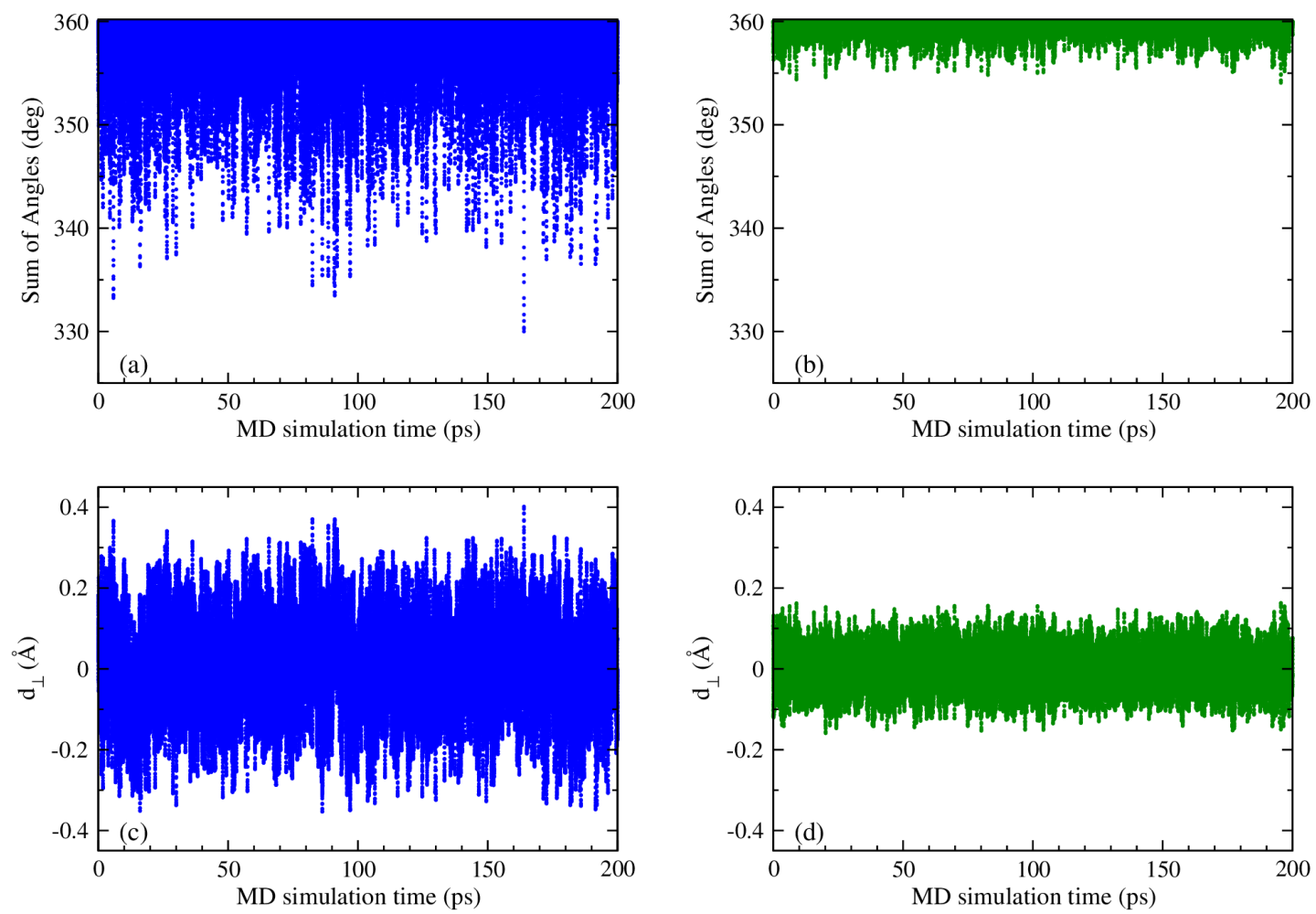

Fig. 4 The sum of angles around NE1 in Trp as a function of simulation time (a) by applying the C36 FF (Trp set 0) and (b) by including an improper torsion function with $K_{\mathrm{imp}}$ of $90 \mathrm{kcal} / \mathrm{mol}^{-\mathrm{rad}^{2}}$ (Trp set 4). The calculated $d_{\perp}$ for NE1 as a function of time (c) by applying the C36 FF and (d) by including an improper torsion function with $K_{\text {imp }}$ of $90 \mathrm{kcal} / \mathrm{mol}^{-\mathrm{rad}^{2}}$. A sum of $360^{\circ}$ and $d_{\perp}=0$ correspond to planarity.
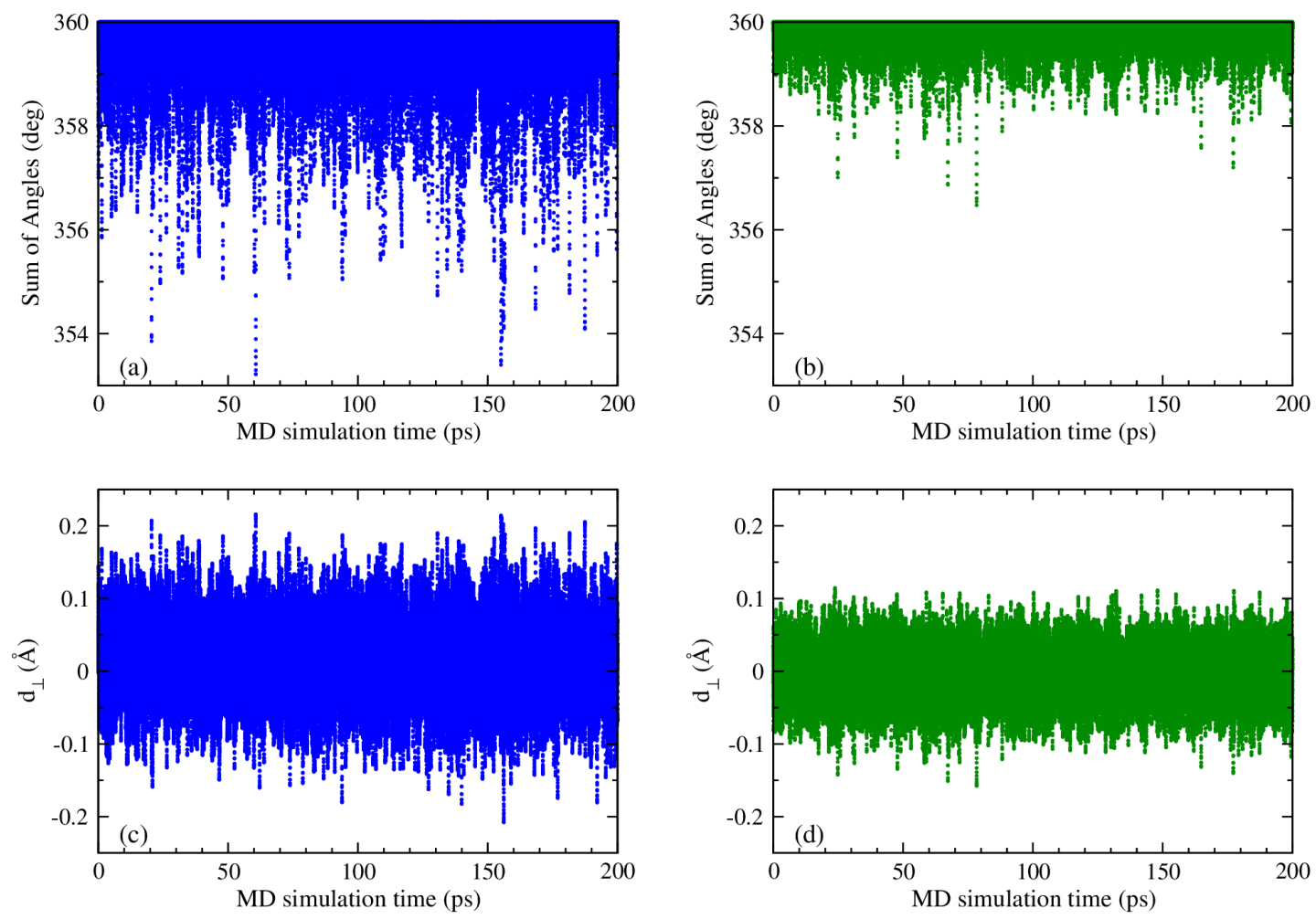

Fig. 5 The sum of angles around CG in Tyr as a function of simulation time (a) by applying the C36 FF (Tyr set 0 ) and (b) by including an improper torsion function with $K_{\mathrm{imp}}$ of $90 \mathrm{kcal} / \mathrm{mol}^{-\mathrm{rad}^{2}}$ (Tyr set 4). The calculated $d_{\perp}$ for $\mathrm{CG}$ as a function of time (c) by applying the C36 FF and (d) by including

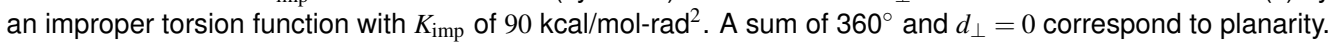




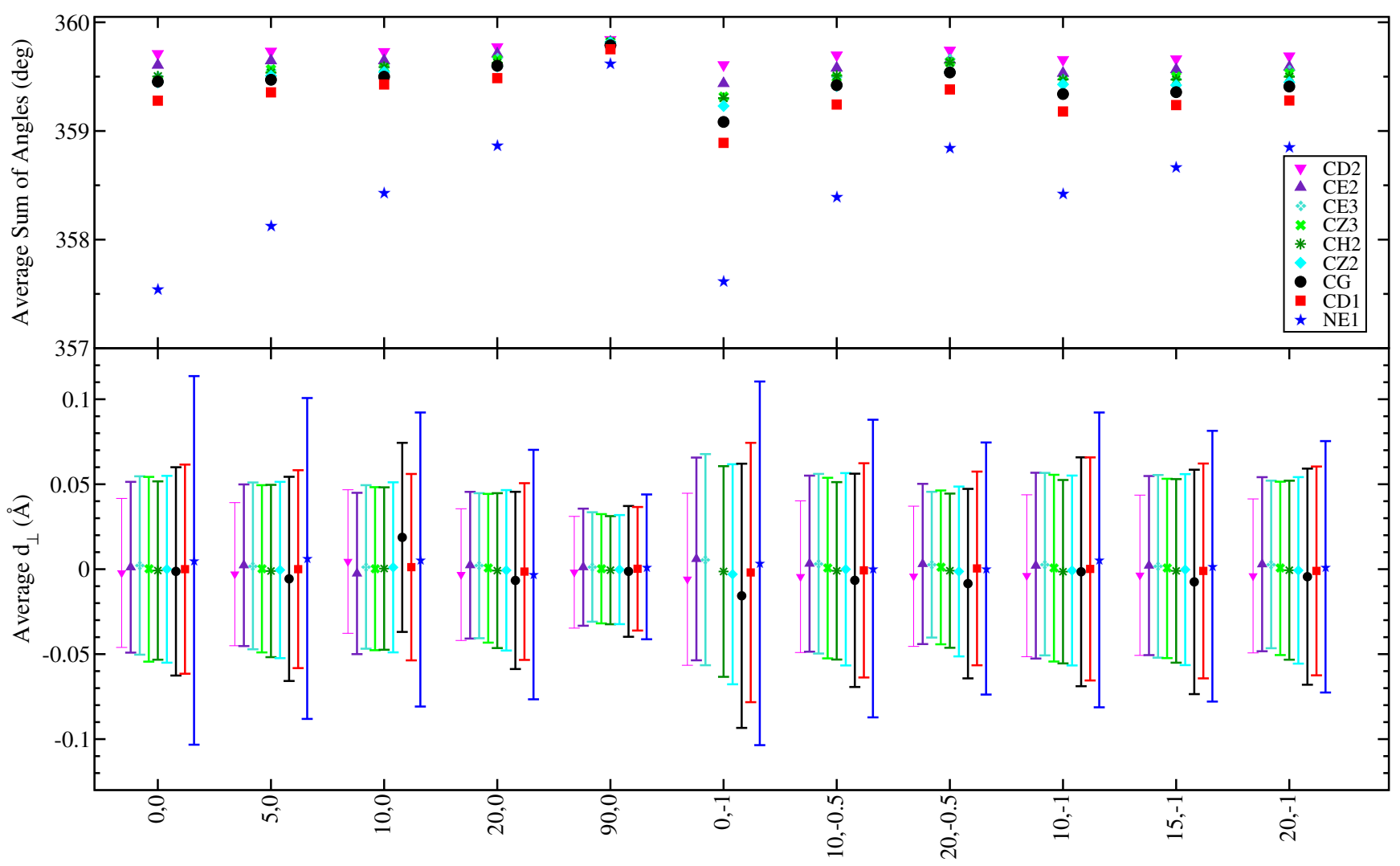

Fig. 6 Variability in the average sum of bond angles and the average $d_{\perp}$ for atoms in the rings of Trp over a 200 ps simulation for each FF set. $\mathrm{x}$-axis labels are $K_{\mathrm{imp}},\left\{K_{\mathrm{tor}}-K_{\mathrm{tor}}^{C 36}\right\}$; atom labels in the legend are taken from Fig. 1

more planar in comparison to simulations using the C36 FF. The overall geometric analysis implies that including an improper torsion potential term reduces the nonplanarity of the aromatic ring by limiting out-of-plane fluctuations that were enhanced by reducing $K_{\text {tor }}$.

The geometric results of NVE MD simulations of zwitterionic Trp and Tyr are compared to NPT MD simulation results in Figs. S6 and S7 ESI $\dagger$. The similarities between NVE and NPT averages confirm that the temperature and pressure control algorithms applied in NPT MD simulations hove not affected the outof-plane motions of atoms in the rings of these two molecules.

Quantum mechanics calculations for D-Trp show sums of $360^{\circ}$ for all ring atoms in a conformer with an optimized structure. 39 Interior angle sums of $720^{\circ}$ for the phenyl ring and $540^{\circ}$ for the pyrrole ring in that work are also consistent with a planar geometry. Quantum mechanics calculations on L-Trp $45 / 46$ do not report enough angles for comparisons to be made around each ring atom. For the benzene ring in L-Trp, Leyton et al. ${ }^{46}$ report interior bond angles that sum to $719.7^{\circ}$, just below the $720^{\circ}$ of a planar hexagon. It is not clear if the $0.3^{\circ}$ difference is significant or is a consequence of rounding. Cao and Fischer ${ }^{45}$ report interior angles that sum to $719.99^{\circ}$ for this ring. Bond angles reported in quantum mechanics calculations on Tyr 41 indicate sums of $360^{\circ}$ for angles around five of the ring carbon atoms, with the sixth reaching $360.01^{\circ}$.

\section{Impacts of Nonplanar Geometry}

The out-of-plane deviations quantified in Figs. 6 and 7 have potential impacts on the biological activity of Trp and Tyr. Largerscale vibrations of ring atoms affect the ability of other molecules to reside nearby. While sub-Ångstrom differences are small on an absolute scale, they can impact the concentration and proximity of the closest molecules. To investigate this issue, we calculated the radial distribution function $g(r)$ between atoms in the rings of Trp or Tyr and atoms of water molecules on the basis of 200 ps MD simulation results by using VMD ${ }^{64}$ software. Changes in outof-plane fluctuations affected the position of the closest neighbor shell. For example, Fig. 8 shows $g(r)$ between the NE1 atom of Trp and the hydrogen atoms of water molecules for three different FF sets. Differences in $g(r)$ over a plateau around $r \approx 3.7 \AA$ to $5.5 \AA$ confirm that applying different FFs affects the probability of water molecules that are closest to atom NE1. We found the same effect to a greater extent for atoms in the ring of Tyr, as shown in Fig. 9 for $g(r)$ between the CD1 atom of Tyr and oxygen of water molecules for three different FF sets. Hence, larger vs. smaller out-of-plane vibrations of heteraromatic rings impact interactions with other nearby molecules. Examples include the effective size of a ring as it shapes an active site or inserts into a membrane.

\section{Out-of-Plane Dynamics}

The dynamics of out-of-plane distance fluctuations within simulated structures were considered next. The autocorrelation func- 


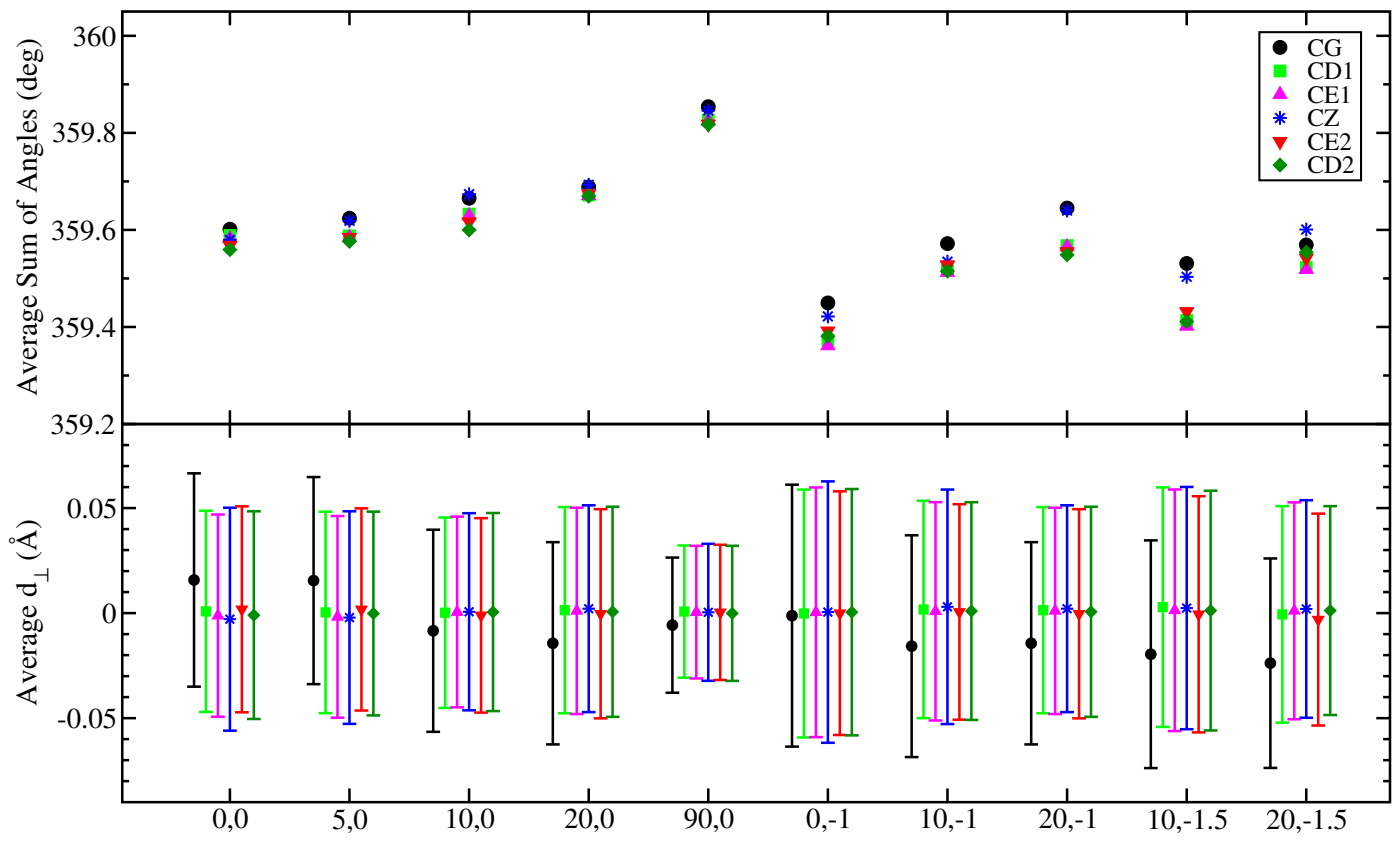

Fig. 7 Variability in the average sum of bond angles and the average $d_{\perp}$ for atoms in the ring of Tyr over a 200 ps simulation for each FF set. $\mathrm{x}$-axis labels are $K_{\mathrm{imp}},\left\{K_{\mathrm{tor}}-K_{\mathrm{tor}}^{C 36}\right\}$; atom labels in the legend are taken from Fig. 1

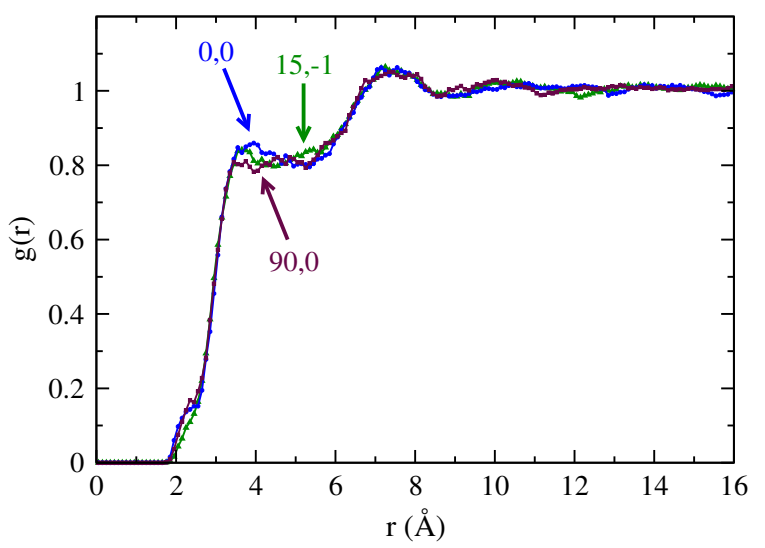

Fig. $8 \mathrm{NE} 1(\operatorname{Trp})-\mathrm{H}\left(\mathrm{H}_{2} \mathrm{O}\right)$ radial distribution function on the basis of three different FF sets.

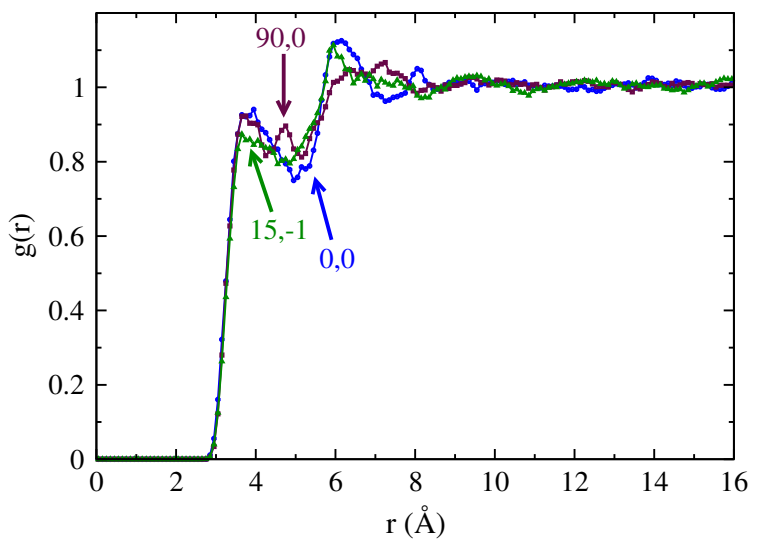

Fig. $9 \mathrm{CD} 1(\mathrm{Tyr})-\mathrm{O}\left(\mathrm{H}_{2} \mathrm{O}\right)$ radial distribution function on the basis of three different FF sets. tion $C(\tau)$ of $d_{\perp}$ (eq 3) was first calculated for each FF set listed in Table 2 Though the full range of time separations was computed, only the first 2 ps are shown because that encompasses the full relaxation to zero. To convert calculated time domain results of $C(\tau)$ into the frequency domain, Fourier transformation (FT) was then performed on the $C(\tau)$ results. A 10-point moving average was applied to the frequency domain results. ${ }^{66}$ Figs. 10 and S8 (ESI. $\dagger$ ) provide $C(\tau)$ and 10-point moving averages after FT for planes centered on atoms NE1 and CE3, respectively, of the aromatic ring of Trp. Figs. 11 and S9 (ESI. $\dagger$ ) provide analogous results centered on CG and CZ of Tyr, respectively. Separate rows in these figures show results for different FF sets.

As implied in Fig. 10a, the relaxation time decreases when improper torsions are included in C36. Applying the largest improper torsion parameter induced an oscillation of period $\sim 1 / 5$ ps. When improper torsion terms were included, the NE1, CD1, HE1, and CE2 atoms of Trp tended to spend shorter periods of time away from a planar structure than without improper torsion contributions. In Fig. 10p, each peak shows frequencies that correspond to out-of-plane displacements of these atoms in the rings. Although $d_{\perp}$ represents the distance of the central atom from the plane containing three connected atoms, this does not mean that only the central atom is moving while the other three connected atoms do not move during an MD simulation. Instead, frequencies obtained from each FT correspond to out-of-plane displacement vibrations of all four connected atoms for which $d_{\perp}$ has been calculated. These out-of-plane vibrational peaks shifted to higher frequencies after including the improper torsion term for these four atoms. An improper torsion potential controls only outof-plane motions of atoms. While increasing $K_{\text {imp }}$ decreases outof-plane displacements of atoms in the aromatic rings, the speeds of these displacements increased as a result, and the frequencies 
(a) $C(\tau)$
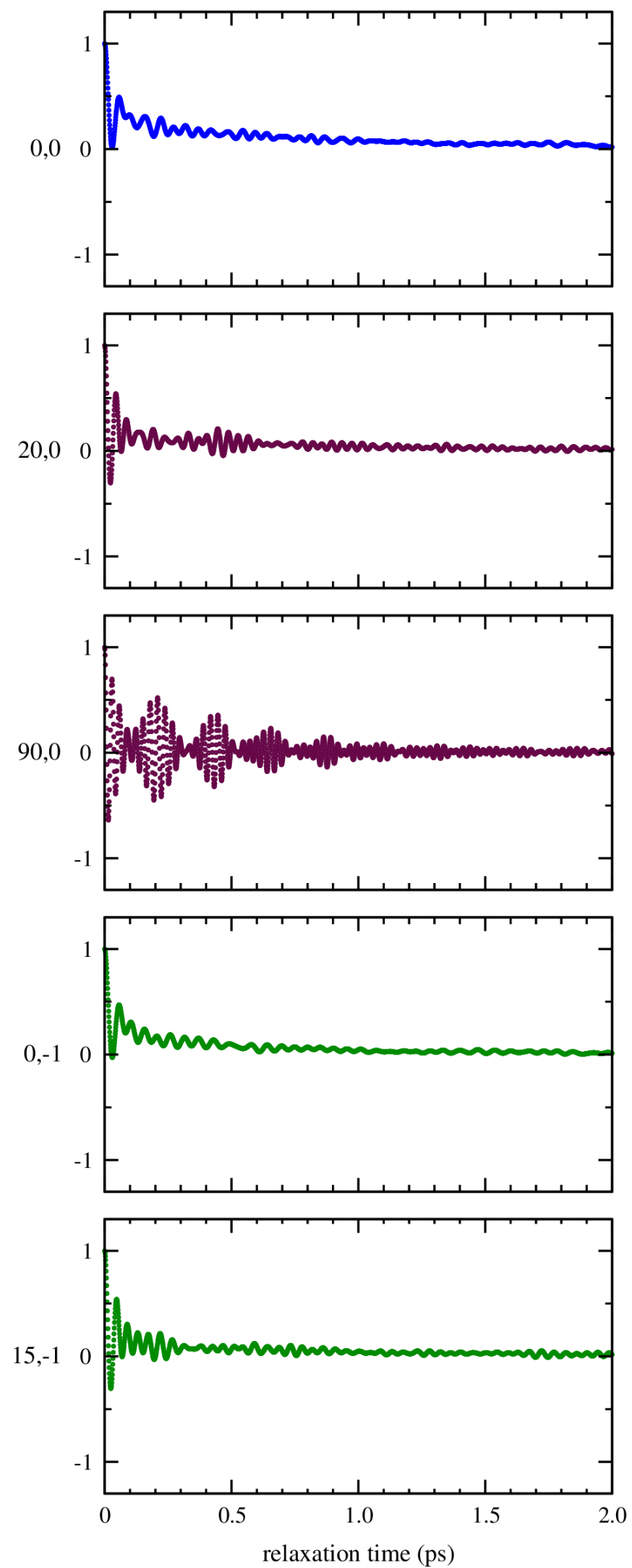

(b) $\mathrm{FT}[C]$
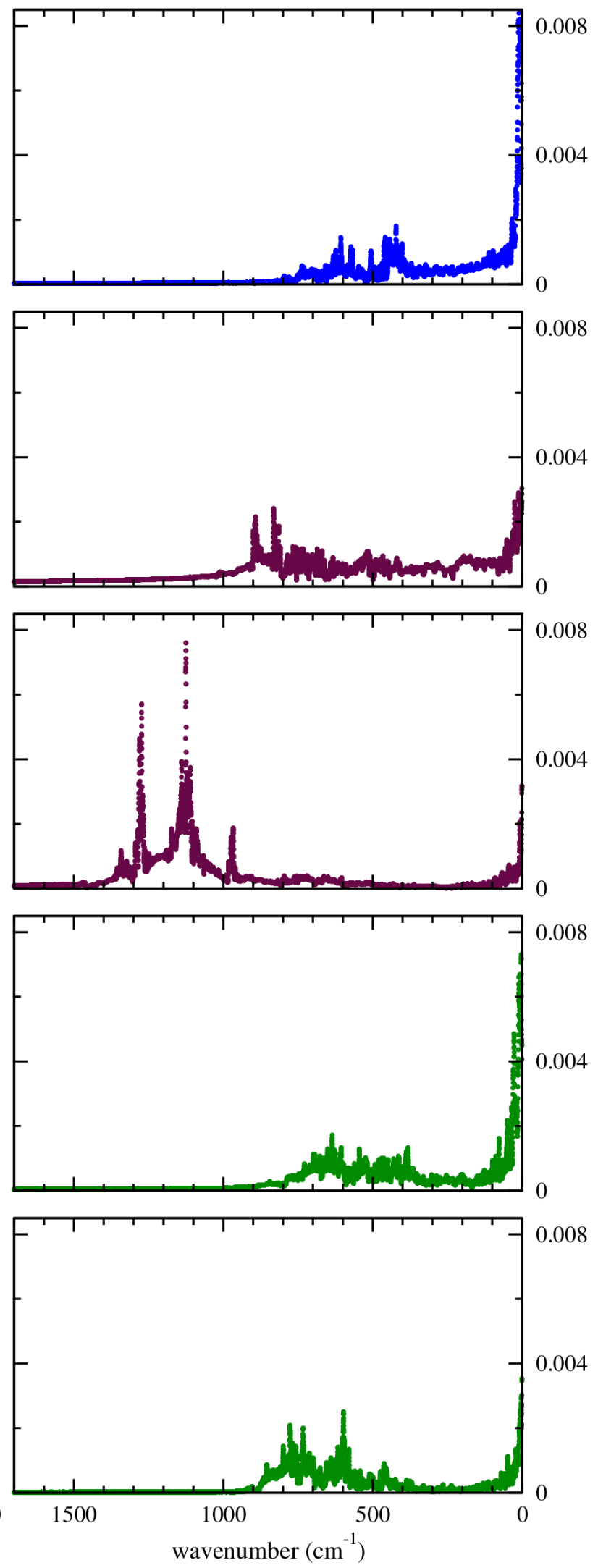

Fig. 10 (a) The time autocorrelation function of $d_{\perp}$ and (b) its Fourier transform for atoms NE1, CD1, HE1, and CE2 of Trp under some FF sets. 
(a) $C(\tau)$
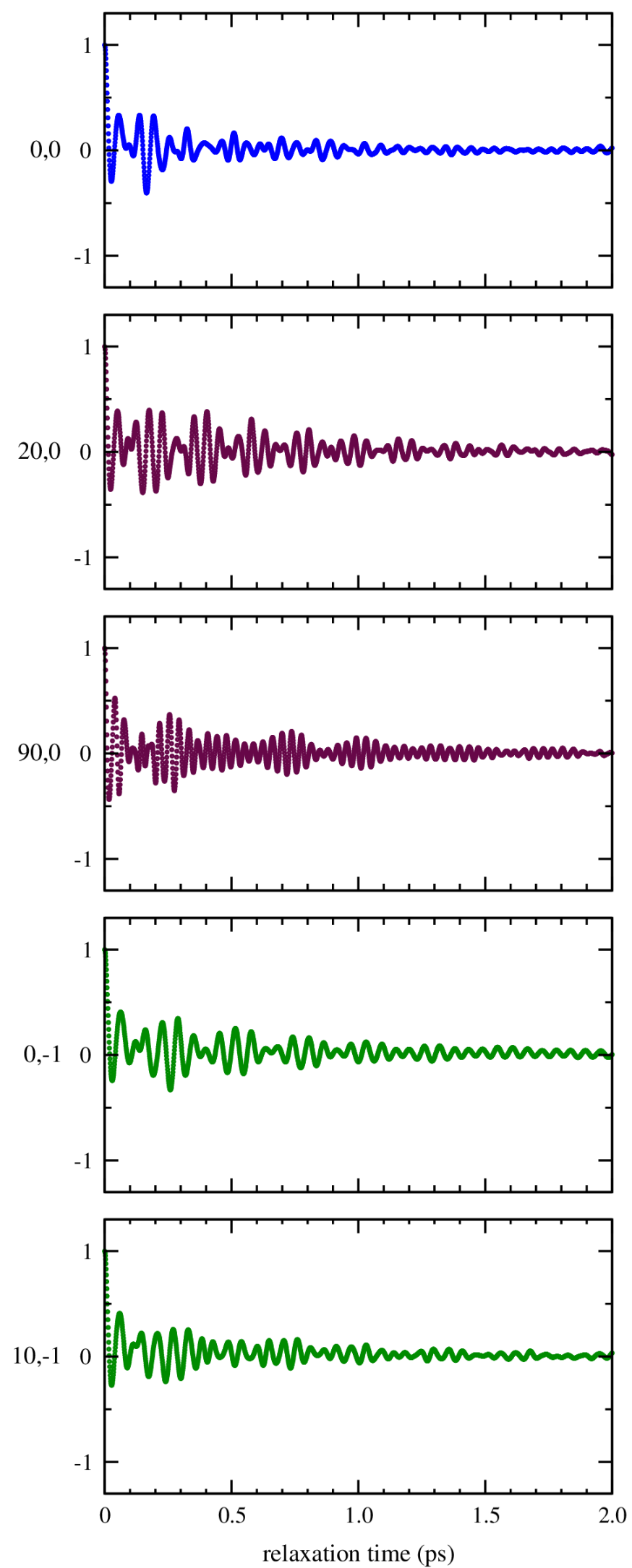

(b) FT[C]

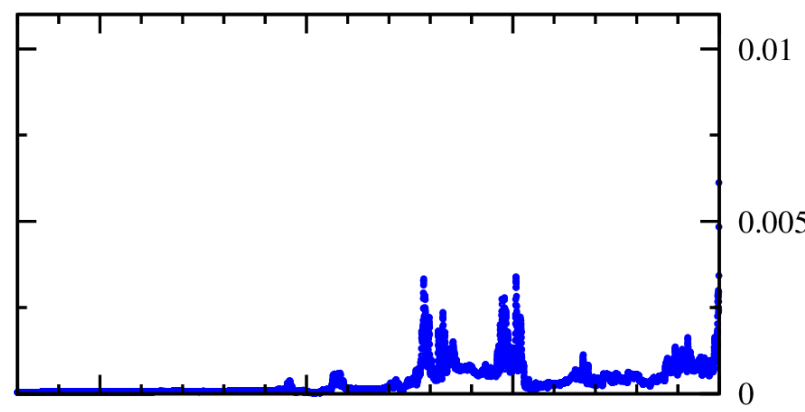

0.01
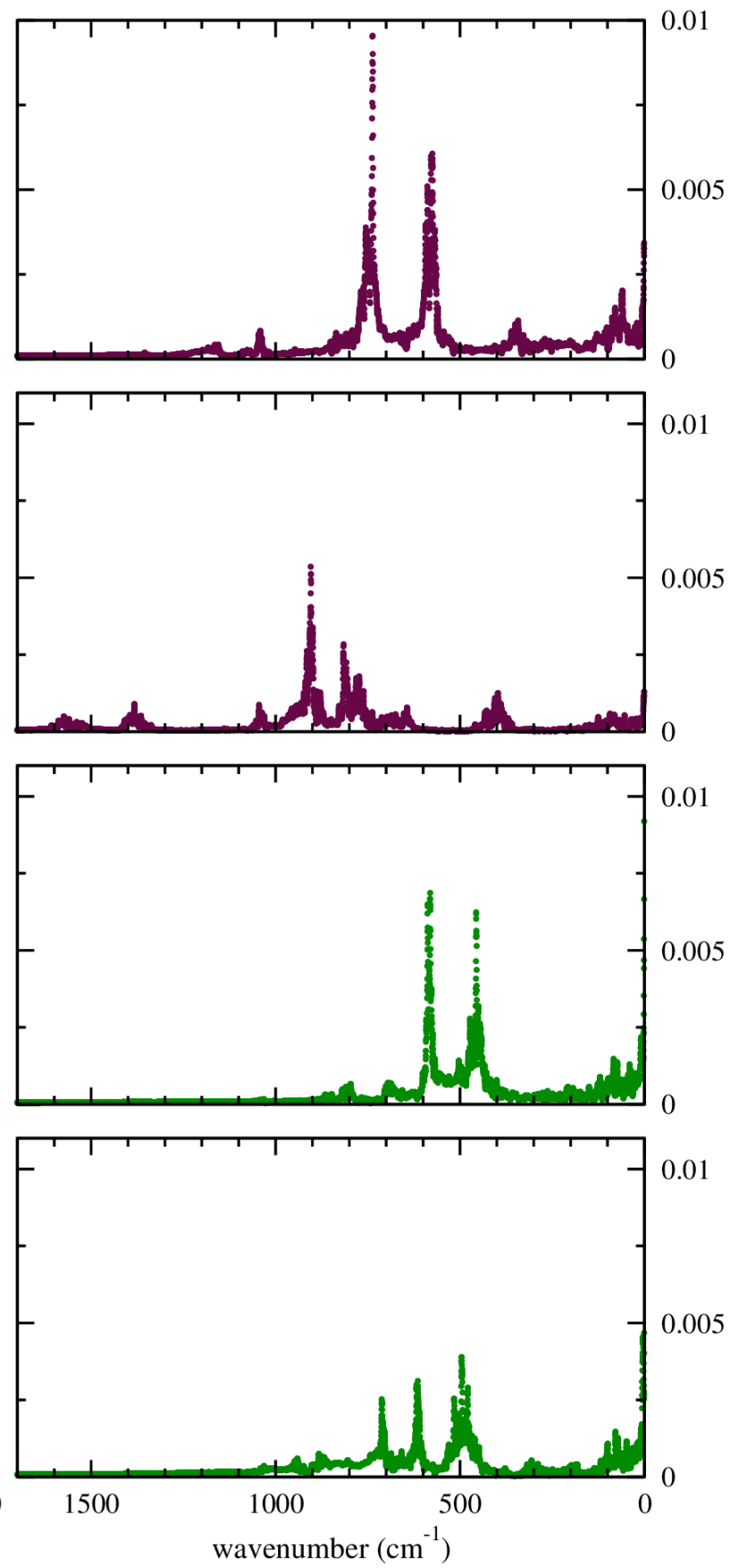

Fig. 11 (a) The time autocorrelation function of $d_{\perp}$ and (b) its Fourier transform for atoms CG, CD1, CD2, and CB of Tyr under some FF sets. 
of these vibrations increased. For the $(0,-1)$ FF set, which corresponds to changes only in the torsion angle potential, neither a significant difference in the relaxation time nor a shift to lower frequencies was seen. Upon adding the improper torsion term to this FF set, such as in case $(15,-1)$, the relaxation time decreased and thus shifted the out-of-plane vibrations to higher frequencies. For atom CE3 (as a central atom), surrounded by CD2, CZ3, and HE3, the relaxation time does not change significantly for each different FF set (Fig. S84, ESI $\dagger$ ). However, Fig. S8p (ESI $\dagger$ ) shows that out-of-plane vibrations shifted to higher frequencies as the improper torsion force constant increased. For the $(0,-1)$ FF set, peaks shifted to lower frequencies compared to the C36 FF because the torsion angle force constant had decreased. For the $(15,-1)$ FF set, the peaks shifted back toward higher frequencies. For example, the peak around $1000 \mathrm{~cm}^{-1}$ in the C36 FF set was shifted to around $950 \mathrm{~cm}^{-1}$ in the $(15,-1)$ FF set. Results with the C36 FF are comparable for methylamide terminal groups (Figs. S2 , $S 2 \mathrm{~d}$ in ESI $\dagger$ ) and for zwitterionic Trp in NVE MD simulations (Figs. S4, S4d in ESI $\dagger$ ).

Similarly, out-of-plane displacements among central atom CG and neighbors CD1, CD2, and CB (Fig. 11) and among atoms CZ, $\mathrm{CE} 1, \mathrm{OH}$, and CE2 (Fig. S9. ESI $\dagger$ ) of Tyr shifted to higher frequencies after including an improper torsion term. In both cases, when employing the $(0,-1) \mathrm{FF}$ set, peaks shifted a little to lower frequencies compared to the C36 FF. When employing the $(10,-1)$ FF set, the peaks remained around the same frequencies as produced by the C36 FF. Results with the C36 FF show similar frequencies for methylamide terminal groups (Figs. S3, S3 in in ESI $\dagger$ ) and for zwitterionic Tyr in NVE MD simulations (Figs. S5, S5 $\mathrm{d}$ in ESI $\dagger$ ).

\section{Out-of-Plane Normal Modes}

For both Trp and Tyr, the out-of-plane displacements suggest that multiple force field parameterizations enable an amino acid to exhibit the same vibrational frequencies yet different fluctuations from planarity. These results also indicate that although including improper torsions for atoms in the rings of Trp and Tyr contribute to atoms being in a planar ring structure, including improper torsions terms and changing torsion angles around these atoms also have direct effects on the out-of-plane vibrations and dynamics of atoms in the rings. Out-of-plane vibrations of a single atom also arise at multiple FF-dependent frequencies.

The effects of improper torsion and torsion angles on the dynamics and vibrations of atoms encouraged us to investigate the effects of different FF sets on atomic vibrations in more detail by applying all-atom normal mode analysis (NMA), which provides dynamics information to complement the geometric analysis of heteroaromatic ring planarity. First, NMA was implemented on the minimized structures of Trp and Tyr in vacuum and then in aqueous solution on the basis of the C36 FF to find the effect of water molecules on the vibrational modes of Trp and Tyr. Our results, shown in Fig. 12 and discussed below, indicate that wateramino acid interactions did not impose important changes on the vibrations of the atoms within the side chain aromatic rings. Hence, NMA was applied to individual Trp or Tyr configurations over MD simulations of aqueous solutions that were conducted using the different FF sets. NMA results were compared to prior Raman (RS) and infrared (IR) spectroscopy $43 / 44 / 46 / 49$ and quan-

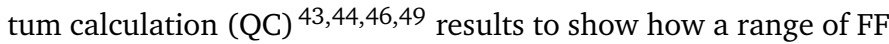
set modifications affect atomic vibrations in heteroaromatic rings while simultaneously altering the planarity of such rings. Geometric analysis of individual modes is discussed in $\mathrm{ESI} \dagger$.

\section{Effects of water molecules on normal modes}

To check whether applying NMA on an individual amino acid molecule is sufficient to simulate out-of-plane motions, compared to NMA on the entire amino acid/water system, NMA was applied on the minimized structures of Trp and Tyr in both vacuum and in aqueous solution using the C36 FF. Fig. 12 and Fig. 12 p provide results for Trp and Tyr, respectively. In each graph, the upper panel shows the frequencies that were obtained for the minimized structure of each aromatic amino acid. These modes were considered as reference modes for the C36 FF. There are $3 N-6=75$ and 66 normal modes calculated for the Trp and Tyr molecules, respectively. Visualizations indicated some backbone/linker vibrational modes centered away from the rings of each amino acid (such as on the ammonium group, alpha carbon, carboxylate group, or linker beta carbon of Trp or Tyr) in which atoms in the aromatic rings did not move. For clarity, only frequencies that correspond to vibrational motions that include atoms in the aromatic rings are presented. Some vibrational modes that are presented in the upper panels of Fig. 12 included combined motions of atoms in the linker, backbone, and aromatic rings. Animations that show all calculated vibrational modes for Trp and Tyr in vacuum (on the basis of the C36 FF) are provided in ESI $\dagger$. In addition, all reference modes on the basis of the C36 FF are listed in Tables $\mathrm{S} 2$ and S3 (ESI $\dagger$ ) for Trp and Tyr, respectively.

In the simulated systems of each amino acid with water, there were a large number of vibrational modes in which only the water molecules vibrated. In most of the modes that corresponded to vibrations of the atoms in aromatic amino acids, water molecules also vibrated. Since there were significantly more atoms in water than in Trp or Tyr, water molecule vibrations corresponded to the largest portion of the eigenvectors of these modes. To find similar modes between the vacuum and aqueous systems, the highest dot product of each reference eigenvector and corresponding eigenvectors of the aqueous system needs to be found. To that end, we reduced the eigenvectors of the aqueous system by removing $(x, y, z)$ atom coordinates of the water molecules, leaving only the amino acid components. Then these eigenvectors, which had the same number of elements as the reference eigenvectors, were renormalized and used in the dot product comparisons. As a result, the lower panels of Fig. $12 \mathrm{a}$ and Fig. $12 \mathrm{p}$ provide only the modes in the aqueous system that correspond to vibrations of atoms within the aromatic rings. Vibrational modes in the upper and lower panels of Fig. 12 that are shown with the same color correspond to similar motions. The peak heights for modes in the lower panel of these graphs indicate the value of the highest dot product; a higher dot product indicates more similarity between the two vibrational modes. In some cases, especially at frequencies less than $1000 \mathrm{~cm}^{-1}$, a single reference mode in vacuum was 
found to have several similar vibrational modes in the aqueous system. This indicates that some modes of Trp or Tyr are distributed over a closely related range of frequencies in the aqueous system. It can be seen in Fig. 12 that the frequency distribution of each mode in the aqueous system was centered around the frequency of its corresponding reference modes. Through watching animations of these distributed aqueous modes, we found that water molecules have vibrations that are mainly caused by their interactions with each other. Coincidentally, some modes in isolated Trp and Tyr heteroaromatic rings have the same frequencies as modes in the aqueous systems that include combinations of both water vibrations and Trp or Tyr vibrations.

We have not only relied on the results of dot product calculations to find similar modes to the C36 reference modes, especially in cases where all the atoms in Trp or Tyr rings were moving together. In some modes, more participation came from atoms in the zwitterionic portions of Trp and Tyr than from atoms in the aromatic rings. Although the dot product was high enough for such modes, similarity of vibrational motions within the aromatic ring cannot be demonstrated; essentially they are backbone/linker modes with small ring contributions. Hence, in these cases, similar modes were chosen solely on the basis of observed movements of ring atoms in the animations.

In aqueous systems, charged ammonium and carboxylate groups have significant nonbonded interactions with water molecules, such as hydrogen bonds shown in Fig. 3. These hydrogen bonds were identified by applying the default PyMOL 65 hydrogen bond criteria: $3.6 \AA$ for the maximum distance of ideal hydrogen bonds and $63^{\circ}$ for the maximum angle of a marginal hydrogen bond. These nonbonded interactions, which do not exist in the vacuum systems, clearly affect vibrational motions of atoms in these functional groups. Consequently, reference modes that include vibrations of these ionic groups become distributed over some frequencies in the aqueous system. Specific modes that correspond only to motions of these end groups are not included in Fig. 12 In some modes of Trp and Tyr, atoms in both the zwitterionic portion and the aromatic rings were vibrating. The nonbonded interactions of ionic groups in these modes in aqueous systems could be one cause that affects these frequencies.

The vibrational frequencies of ring atoms in the Trp or Tyr that have a high electronegativity (such as NE1) can also be affected by nonbonded interactions with water molecules. For example, there is a bond stretching vibrational mode for the nitrogen atom and the attached hydrogen atom (HE1) in the indole ring. Our results with C36 FF find this mode at a frequency of $3680 \mathrm{~cm}^{-1}$ for the vacuum and $3655 \mathrm{~cm}^{-1}$ for the aqueous systems, respectively. In the aqueous system, HE1 forms a hydrogen bond with an oxygen atom of a water molecule (Fig. 3). This shift to lower frequency is qualitatively consistent with experiment. Raman and infrared spectroscopy studies of pyrrole in bulk and in carbon tetrachloride solution found that the NH stretching band shifted $93 \mathrm{~cm}^{-1}$ lower when NH groups self-associated by hydrogen bonding in solution. ${ }^{67}$ Here we have found a shift of $25 \mathrm{~cm}^{-1}$ lower due to hydrogen bonding to water in our simulations.

In summary, consideration of water molecules in NMA calculations does not significantly change the frequencies of modes that correspond to vibrations of atoms in the aromatic rings of Trp and Tyr. The dot products of each reference eigenvector and the eigenvectors of the solution system indicate that vibrational modes of atoms in the aromatic rings occur at similar frequencies. For both systems, some of these modes are distributed in the solution system around their reference frequency in a vacuum system (Fig. 12). The main effects of water molecules on normal modes involve zwitterion vibrations, rather than out-of-plane vibrations. These results support our hypothesis that applying NMA by using individual Trp or Tyr configurations within MD simulations of aqueous solutions does not significantly affect out-of-plane vibrational frequencies of atoms within the aromatic ring.

\section{Effects of different force fields on vibrations}

NMA was applied separately on single minimized structures of Trp and Tyr zwitterions for each FF set, and the obtained eigenvectors were considered as reference modes. Fig. 13 and Fig. 14 show the vibrational frequencies of these reference modes for some FF sets. Reference modes that include out-of-plane vibrations are indicated by taller red bars and by letters in Tables S2 and S3. ESI $\dagger$. Only these were affected by changes in $K_{\text {imp }}$ and $K_{\text {tor }}$. Modes that contain in-plane vibrations of atoms in the rings are shown by shorter bars of other colors. Some normal modes contained out-of-plane and planar vibrations; these are indicated by using both bars and by underlining their labels in Tables S2 and S3. Frequencies of modes that did not contain out-of-plane components remained unchanged across these FF sets. One case for Trp (mode 45, Table S2, ESI $\dagger$ ) showed a very small HE3 outof-plane fluctuation amidst vibrations dominated by planar and backbone/linker motions. This mode was not labeled because its out-of-plane component was so small.

To find similar modes between two FF sets, dot products of their reference eigenvectors were calculated. Modes with the highest dot product were identified as similar modes and labeled by the same letters for averaged results shown in Fig. 15 and Fig. 16. Moreover, we watched animations of out-of-plane vibrational modes to confirm and interpret the significance of the dot products.

For each FF set, out-of-plane reference modes enabled finding the frequencies of similar modes throughout each amino acid/water MD simulation. The time average for each out-ofplane mode frequency was calculated over a 200 ps trajectory. There were some modes in which atoms vibrated in the rings, linkers, and backbones of Trp and Tyr. In some of these modes, atomic vibrations in the linker or the backbone were more intense compared to the atomic vibrations in the rings. In these cases, the highest dot product mainly signified atomic vibrations away from the rings. Since we were interested in finding only similar modes that corresponded to out-of-plane vibrations of atoms in the rings, we reduced the dimensions of the eigenvectors by removing the $(x, y, z)$ atom coordinates of the linker and the backbone in the dot product calculations for both Trp and Tyr molecules. The averaged out-of-plane vibrational frequencies for Trp and Tyr are presented in Fig. 15 and Fig. 16, respectively.

To evaluate the FF sets, we compared the calculated out-of- 
(a)

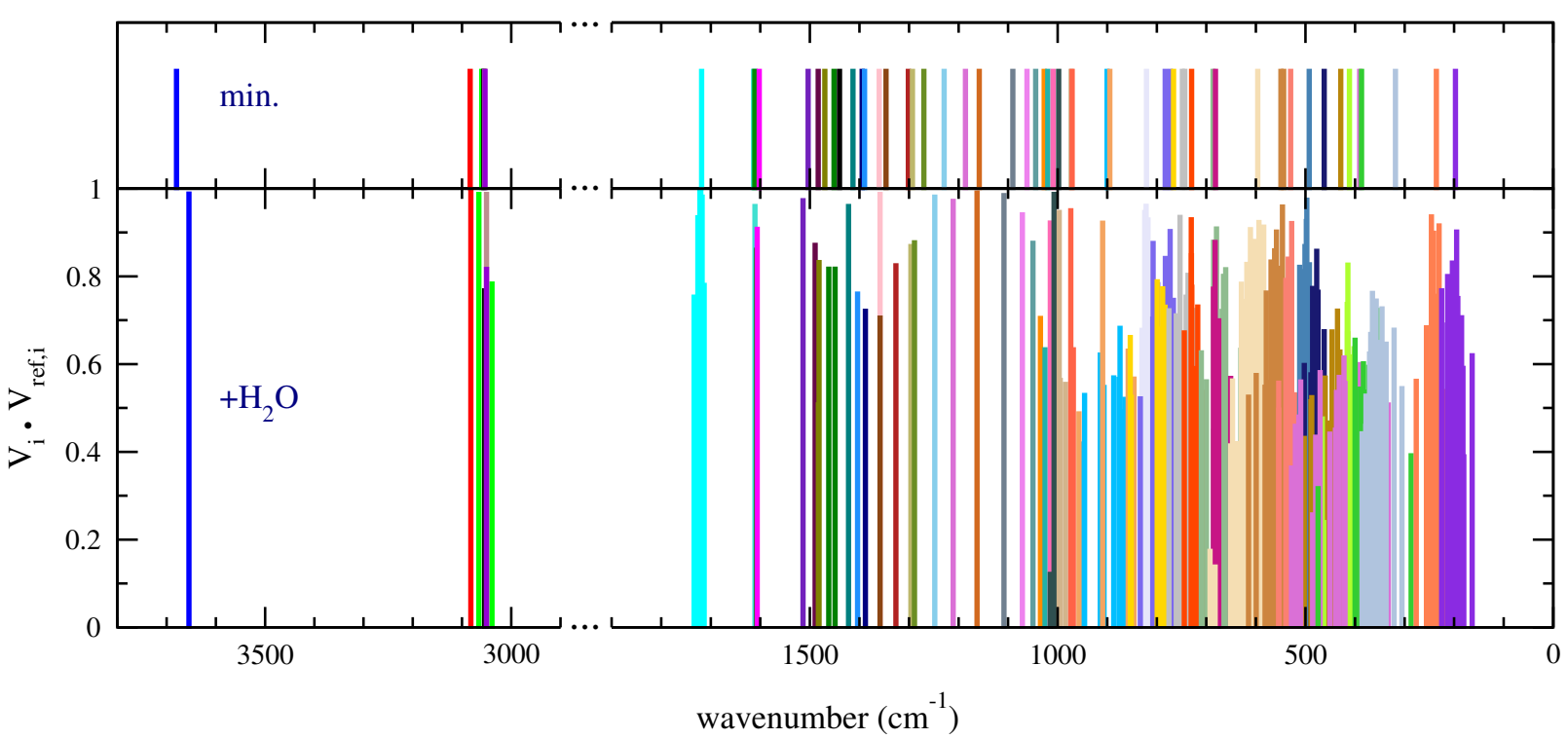

(b)

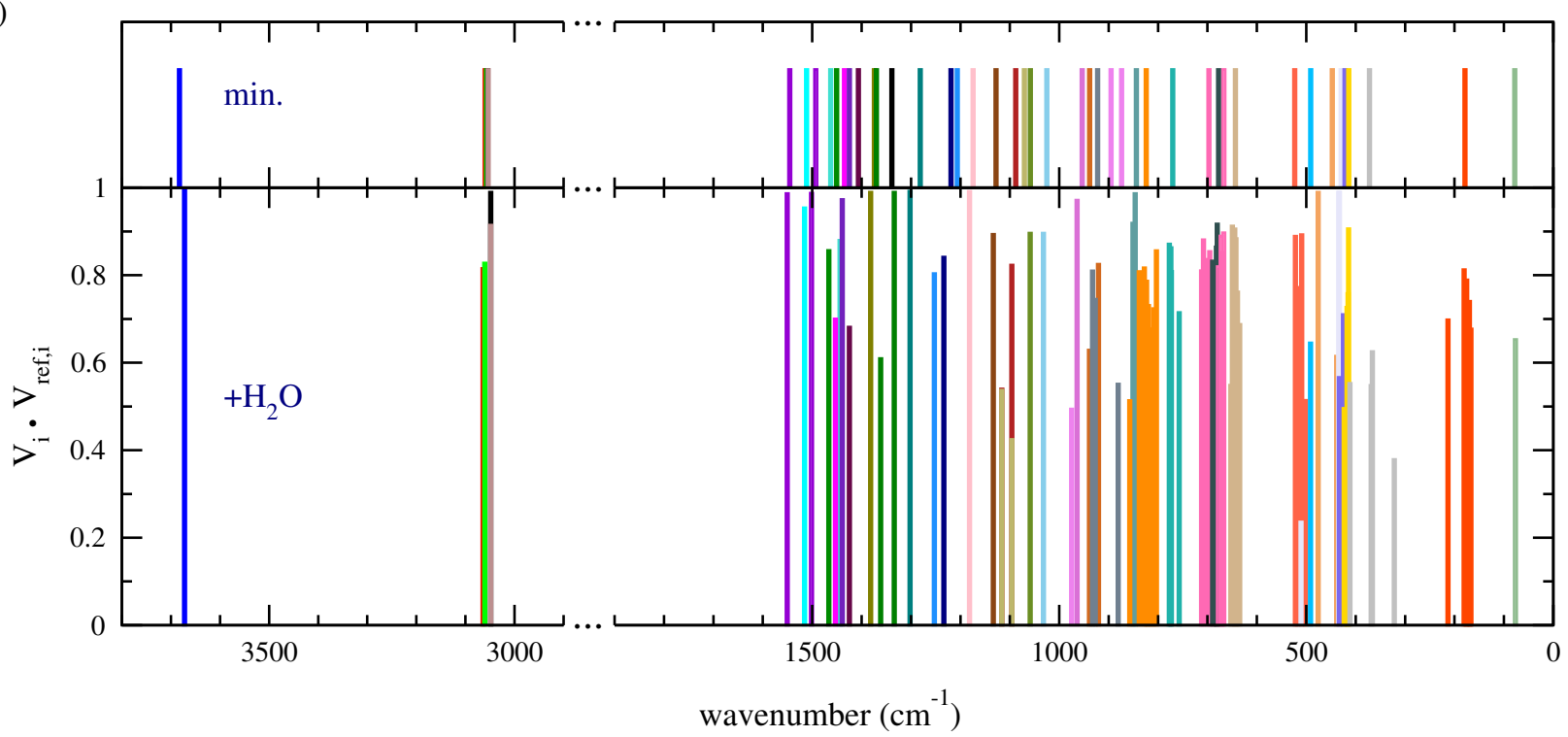

Fig. 12 Calculated normal mode frequencies using the C36 FF that correspond to vibrations of atoms in the rings of (a) Trp and (b) Tyr. Each upper panel shows frequencies from NMA results on a minimized structure of the amino acid (no y-axis units), while each lower panel shows NMA results on an analogous aqueous system ( $y$-axis indicates similarity between modes). Colors for each amino acid indicate modes with similar ring vibrations.

plane frequencies to Raman spectroscopy (RS) and quantum calculation (QC) results obtained from Hernández et al. ${ }^{43}$ They assigned each frequency of the RS to a similar one from QC for frequencies greater than $550 \mathrm{~cm}^{-1}$ for Trp and $650 \mathrm{~cm}^{-1}$ for Tyr. They also described the vibrational motions for each mode using terms such as "out-of-plane bending" and "torsional internal coordinate around the aromatic rings". ${ }^{43}$ This enabled us to compare our calculated frequencies for out-of-plane modes with their results by watching animations of all the vibrational modes, interpreting these motions, and matching the descriptions they reported. These comparative data are presented in the second and third panels of Fig. 15 and Fig. 16 Similar comparisons, though with less clear descriptions available, are possible with the IR data shown in the first panels $\frac{46 / 49}{}$ and with RS and QC results from other literature $\sqrt{44 / 46 / 49}$ at lower frequencies.

\section{Vibrational analysis of tryptophan}

The calculated out-of-plane vibrational modes of Trp on the basis of the C36 FF are presented in Fig. 15 These results were obtained by taking the arithmetic average of wavenumbers for similar modes in aqueous solution along the MD trajectory. Animations of these vibrations, provided in $\mathrm{ESI}^{\dagger}$, were observed and compared to the descriptions of modes in the RS, IR, and QC panels. On the basis of this comparison, the out-of-plane modes of each panel were labeled by letters A-Y. However, there is not a complete match among all vibrations observed in these animations and the descriptions of vibrational modes by Hernández et al., ${ }^{43}$ Leyton et al.,, 46 and Chuang et al. ${ }^{44}$ For instance according to descriptions by Hernández et al., $\frac{43}{4}$ the mode with a frequency 


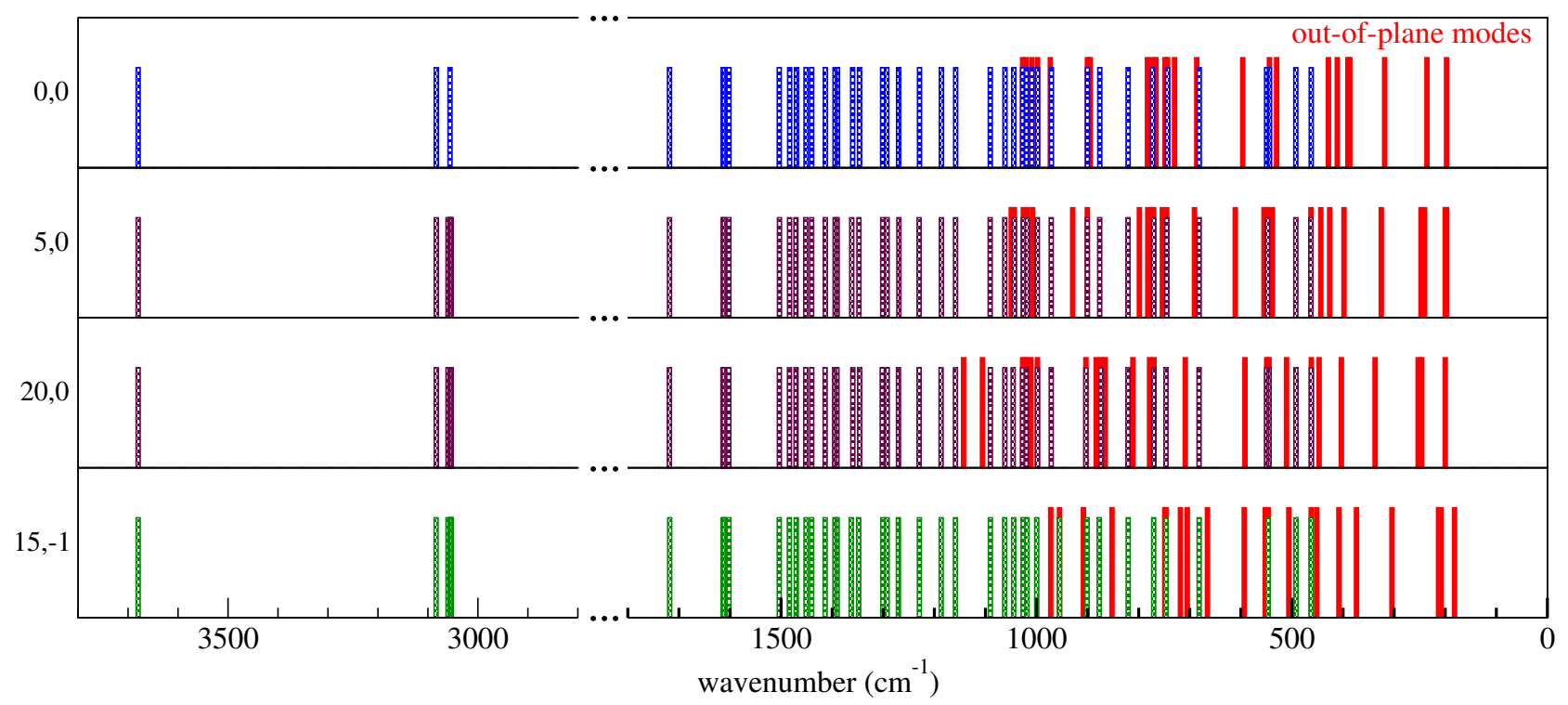

Fig. 13 Reference vibrational frequencies for Trp calculated by NMA on the basis of minimum-energy structures using different FFs, labeled as $\left(K_{\text {imp }}\right.$, $\left.\left\{K_{\text {tor }}-K_{\text {tor }}^{C 36}\right\}\right)$. Only frequencies of modes that include out-of-plane components, indicated by taller red bars, changed for each FF set.

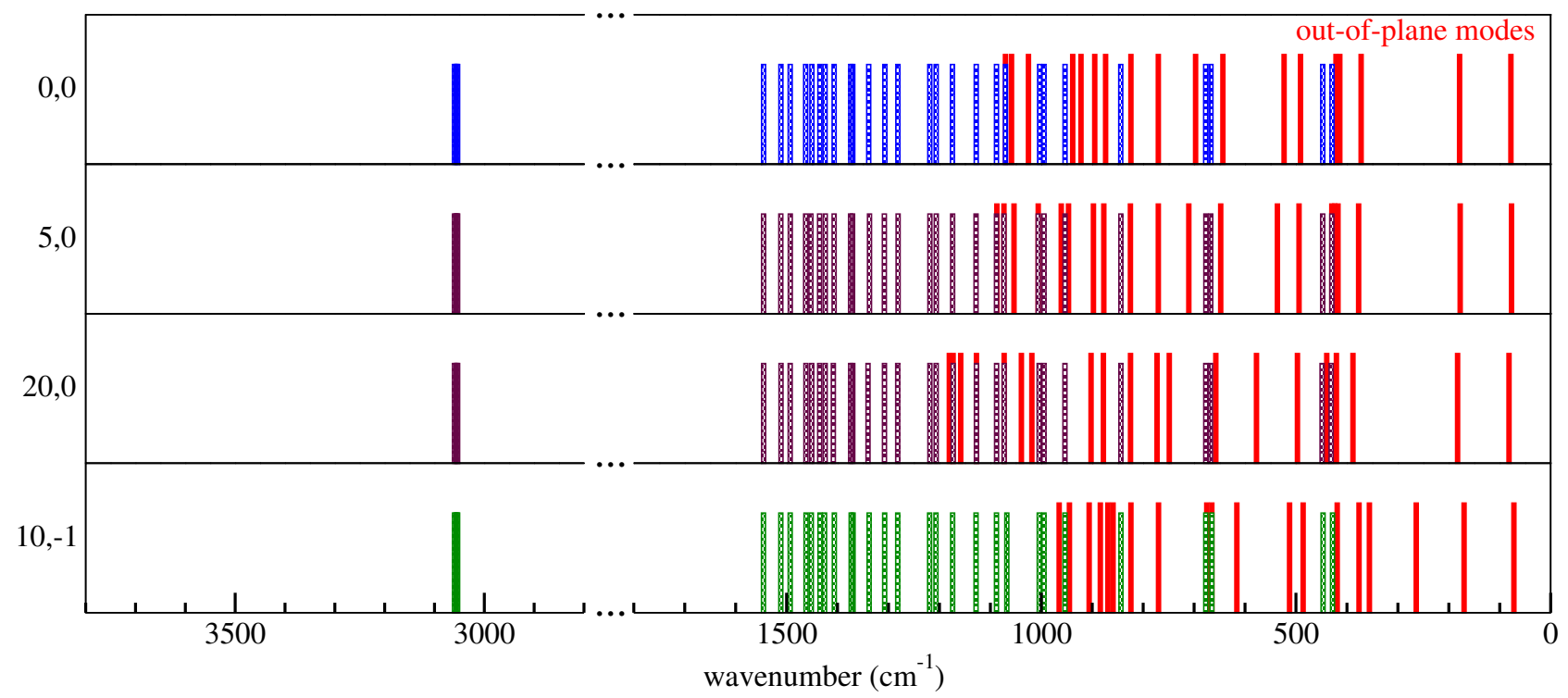

Fig. 14 Reference vibrational frequencies for Tyr calculated by NMA on the basis of minimum-energy structures using different FFs, labeled as ( $K_{\text {imp }}$, $\left\{K_{\text {tor }}-K_{\text {tor }}^{C 36}\right\}$ ). Only frequencies of modes that include out-of-plane components, indicated by taller red bars, changed for each FF set.

of $970 \mathrm{~cm}^{-1}$ in the RS panel $\left(974 \mathrm{~cm}^{-1}\right.$ and $936 \mathrm{~cm}^{-1}$ in the QC panel) corresponds to out-of-plane vibrations of atoms CZ3, CZ2, CE3, CH2, HZ3, HZ2, HE3, and HH2 in the six-membered ring. According to descriptions by Leyton et al., ${ }^{46}$ the mode with a frequency of $966 \mathrm{~cm}^{-1}$ in the RS panel $\left(965 \mathrm{~cm}^{-1}\right.$ in the QC panel or $960 \mathrm{~cm}^{-1}$ in the IR panel) corresponds to an out-ofplane vibration of atoms CZ3 and HZ3. In our animations, a combination of modes U (Movie TRP40, $984 \mathrm{~cm}^{-1}$ ), V (Movie TRP41, $1011 \mathrm{~cm}^{-1}$ ), W (Movie TRP42, $1009 \mathrm{~cm}^{-1}$ ), X (Movie TRP43, $1014 \mathrm{~cm}^{-1}$ ), and Y (Movie TRP44, $1023 \mathrm{~cm}^{-1}$ ) in the C36 panel represents atomic motions that correspond to both of these descriptions. The mode in the RS, IR, and QC panels that is labeled by an asterisk corresponds only to out-of-plane motions of atoms CG, CD1, and HD1. ${ }^{43}$ We could not find a specific mode in the animations of our calculations that presents only this vibration; however, some modes such as M (Movie TRP29, $710 \mathrm{~cm}^{-1}$ ), $\mathrm{N}$ (Movie TRP30, $726 \mathrm{~cm}^{-1}$ ), and O (Movie TRP31, $756 \mathrm{~cm}^{-1}$ ) in our calculations contain vibrations that are similar to that description. Since Leyton et al. 46 and Chuang et al. ${ }^{44}$ have not described modes in as much detail, there are some modes in the RS, IR, and QC panels that we are not completely certain should be considered as out-of-plane modes. These modes are indicated in Fig. 15 by a horizontal-stripe pattern. Some modes could neither be labeled unambiguously nor connected directly to modes (A-I) calculated by NMA because the descriptions in their sources ${ }^{44 / 46}$ only indicate ring torsion and deformation rather than specific 
atom motions.

In some cases, the calculated frequencies and frequency pattern of the out-of-plane modes in the C36 panel differed from the results reported from RS and QC. Out-of-plane vibration of nitrogen in the indole ring was described in a mode with frequency of $578 \mathrm{~cm}^{-1}$ in the RS and QC panels by Hernández et al. ${ }^{43}$ and $628 \mathrm{~cm}^{-1}$ in the RS panel $\left(618 \mathrm{~cm}^{-1}\right.$ and $627 \mathrm{~cm}^{-1}$ in the QC and IR panels) by Leyton et al. $\frac{46}{}$ This vibration appeared in modes $\mathrm{J}$ (Movie TRP24, $543 \mathrm{~cm}^{-1}$ ) and $\mathrm{K}$ (Movie TRP26, $586 \mathrm{~cm}^{-1}$ ) in the C36 panel. These modes also contained some other out-ofplane vibrational modes that were not in the RS and QC results. Mode L (Movie TRP28, $678.5 \mathrm{~cm}^{-1}$ ) obtained by our calculation on the basis of C36 does not exist in the Hernández et al. 43 results. But mode $656 \mathrm{~cm}^{-1}$ in the IR panel $\left(654 \mathrm{~cm}^{-1}\right.$ in the QC panel) by Leyton et al. $\frac{46}{4}$ contains CG and CD1 out-of-plane vibrations that correspond to this vibration. Modes M-R in the C36 panel clustered around the same frequencies as similar modes in the RC and QC panels. Significantly, the frequencies of modes S-Y were higher with C36 compared to these literature sources, such as mode $\mathrm{X}$ at wavenumber $970 \mathrm{~cm}^{-1}$ in the RS panel, $974 \mathrm{~cm}^{-1}$ in the QM panel, and $1014 \mathrm{~cm}^{-1}$ in the $\mathrm{C} 36$ panel.

Instantaneous NMA was also applied on NVE MD simulations of zwitterionic Trp in solution. Almost all out-of-plane motions showed similar frequencies in NPT and NVE MD simulations (Fig S12, ESI $\dagger$ ). Hence, out-of-plane ring atom vibrations were independent of temperature and pressure control algorithms.

Results from NMA on Trp with methylamide terminal groups in solution (Fig. S12, ESI $\dagger$ ) show that most normal mode frequencies and the frequency pattern are similar for both architectures. For most modes, differences between architectures are small compared to the changes that were found upon adding an improper torsion potential and reducing the torsion potential. Frequency results for Trp zwitterion and methylamide terminated Trp architectures differ in similar ways from RS, IR, and QC results.

Including an improper torsion potential and increasing $K_{\mathrm{imp}}$ shifted out-of-plane modes to higher frequencies, thus making discrepancies worse compared to RS, IR, and QC (Fig. 15). In addition, some mode patterns became distributed over a wider frequency range after including the improper torsion potential, such as the frequency distribution for the FF set $(90,0)$ with $K_{\text {imp }}=90 \mathrm{kcal} / \mathrm{mol} \mathrm{rad}^{2}$. Although incorporating an improper torsion potential could reduce the nonplanarity of Trp in an MD simulation, it significantly shifted out-of-plane modes to higher frequencies compared to RS and QC, disturbed the pattern of these modes in the spectra, and increased out-of-plane displacement speeds of atoms in the ring. As a consequence, the frequencies of these modes were increased, and atoms vibrated at higher frequencies compared to their frequencies in Raman spectra. In other words, the frequency $(v)$ increases in a manner similar to that of a simple harmonic oscillator of mass $m$ and stiffness $K$, $v \sim \sqrt{K / m}$. Including an improper torsion potential provides a force constant that directly controls out-of-plane atom vibrations.

The C36 FF showed differences for out-of-plane frequencies compared to the RS, IR, and QC results, even without improper torsion potentials for the aromatic ring of Trp. The differences could be consequences of torsion angle force constants for atoms in the ring. Hence, we investigated how changes in the $K_{\text {tor }}$ affected agreement with RS, IR, and QC for the normal mode frequencies.

Table 2 lists the original $K_{\text {tor }}$ and simultaneous decreases in all ring torsion angle parameters $K_{\text {tor }}$ that were considered. As shown in Fig. 15 for set $(0,-1)$, decreasing the force constants by $1 \mathrm{kcal} / \mathrm{mol}$ for all torsion angles shifted the out-of-plane frequencies lower compared to the C36 FF $(0,0)$, RS, IR, and QC results. Simultaneously, out-of-plane fluctuations increased in magnitude, as shown in the geometric analysis.

Adding an improper torsion potential and weakening the regular torsion potential have countering effects. Thus we tested some FF sets in which the improper torsion potentials were incorporated and the torsion angle force constants were reduced. The out-of-plane vibrational results of these FF sets are shown in Fig. 15. The FF set in which the improper torsion potentials with $K_{\mathrm{imp}}=15 \mathrm{kcal} / \mathrm{mol}_{-\mathrm{rad}^{2}}$ were considered for atoms in the ring of Trp, and all but the smallest $K_{\text {tor }}$ of torsion angles around the aromatic ring were decreased by $1 \mathrm{kcal} / \mathrm{mol}$, provided the best match of both pattern and out-of-plane frequencies compared with the available RS and the QC results. As shown in Fig. 6 this FF set also reduced the out-of-plane displacements of the nitrogen atom in the ring compared to the C36 FF.

NMA results are in agreement with the frequencies obtained by Fourier transformation of time autocorrelation functions for the out-of-plane displacement. Fig. S8 in ESI†presents the effect of different FF sets on the out-of-plane deformation frequencies of atoms CE3, CD2, CZ3, and HE3. Modes V-Y in the $(0,0)$ panel in Fig. 15 are out-of-plane vibrations of these atoms at 1011, 1009, 1014 , and $1023 \mathrm{~cm}^{-1}$, which all have a corresponding wavenumber of $970 \mathrm{~cm}^{-1}$ in the RS panel. Fig. S8 in ESI† shows a sharp signal around $1000 \mathrm{~cm}^{-1}$ for these four atoms in the C36 $(0,0)$ panel. Fig. S8 in ESI†demonstrates that including improper torsion shifts the out-of-plane vibrations of these four atoms to a higher frequency. For example, in FF set $(20,0)$ this peak shifted to around $1140 \mathrm{~cm}^{-1}$. For FF set $(15,-1)$, this peak shifted to around $950 \mathrm{~cm}^{-1}$ with a small shoulder at $970 \mathrm{~cm}^{-1}$, near the corresponding modes (X and $\mathrm{W}$ ) in the RS panel in Fig. 15. A similar interpretation can be applied for atoms NE1, CD1, HE1, and CE2 (Fig. 10 and Fig. 15).

\section{Vibrational analysis of tyrosine}

The results of out-of-plane vibrational modes of Tyr on the basis of the C36 FF (labels A-R) are presented in Fig. 16. The animations of our calculations for Tyr match the out-of-plane vibrational descriptions of RS and $\mathrm{QC}^{43}$ more clearly compared to the Trp results. According to descriptions by Hernández et al., 43 modes at $948 \mathrm{~cm}^{-1}$ in the RS panel $\left(979 \mathrm{~cm}^{-1}\right.$ and $962 \mathrm{~cm}^{-1}$ in the QC panel) correspond to out-of-plane vibrations of atoms CD1, CD2, $\mathrm{CE} 1, \mathrm{CE} 2$, and the hydrogen atoms connected to them. Modes P-R (Movies Tyr38, 40, and 39) in the C36 panel have similar out-ofplane vibrations at $1030.5,1038.4$, and $1052.1 \mathrm{~cm}^{-1}$ and were assigned to these modes. Modes L-O include similar out-of-plane vibrations, and they clustered around 806,830 , and $850 \mathrm{~cm}^{-1}$ in the RS panel and 831, 850, and $864 \mathrm{~cm}^{-1}$ in the QC panel.43 Corresponding modes for C36 are L (Movie TYR31, $857 \mathrm{~cm}^{-1}$ ), N 


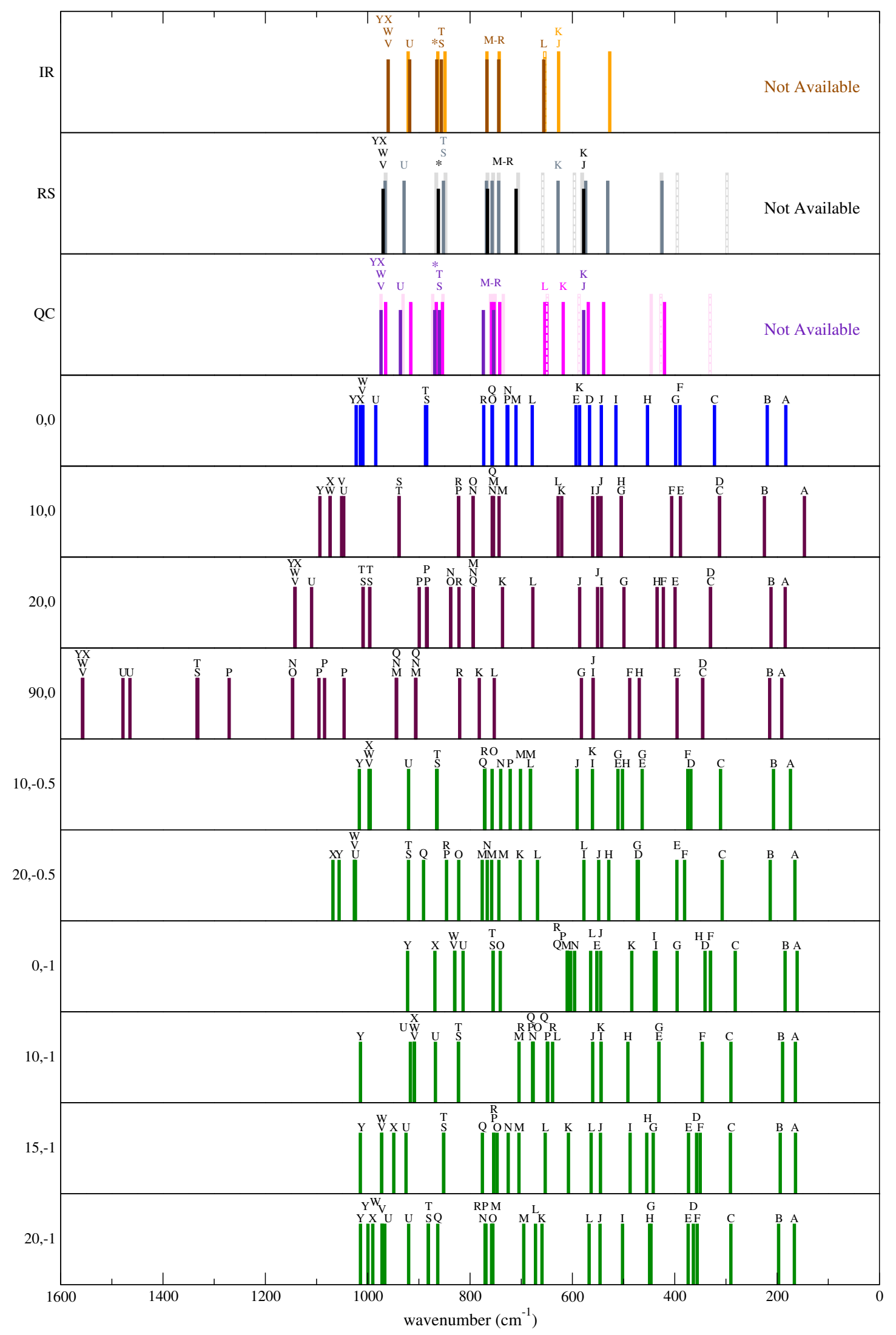

Fig. 15 Normal mode frequencies for Trp. All peaks in all panels present out-of-plane frequencies of Trp. The first panel presents prior infrared spectra (IR) in solution (brown) and solid phase (orange) of Trp. ${ }^{46}$ The second panel presents prior Raman spectra (RS) of Trp in solution (black ${ }^{43}$ and grey ${ }^{46}$ ) and solid phase (light-grey). ${ }^{44}$ The third panel presents prior quantum calculations (QC) of $\operatorname{Trp}+7 \mathrm{H}_{2} \mathrm{O}$ (indigo) ${ }^{43}$, Trp in solution (magenta), ${ }^{46}$ and solid phase (pink). ${ }^{44}$ Other panels show out-of-plane vibrational modes from NMA for each FF set, labelled as $\left(K_{\text {imp }},\left\{K_{\text {tor }}-K_{\text {tor }}^{C 36}\right\}\right)$ and averaged using MD of aqueous solution over 200 ps. Similar modes are shown with the same letter. Not Available indicates ranges in which no vibrations were reported in the cited literature. 


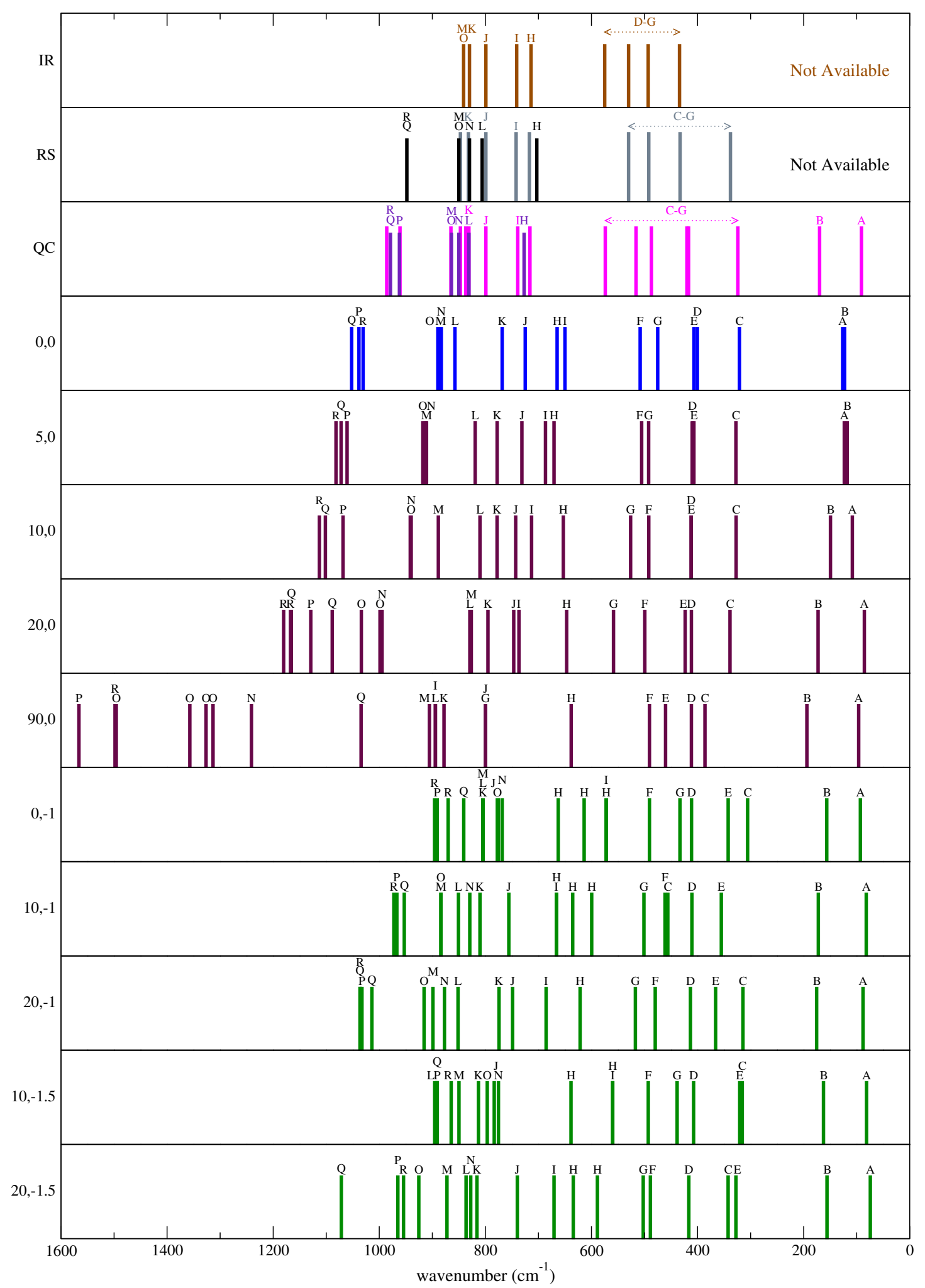

Fig. 16 Normal mode frequencies for Tyr. All peaks in all panels present out-of-plane frequencies of Tyr. The first panel presents prior solid phase infrared spectra (IR, brown) of Tyr. ${ }_{49}^{49}$ The second panel presents prior Raman spectra (RS) of Tyr in solution (black ${ }^{43}$ and grey ${ }^{49}$ ). The third panel presents prior quantum calculations $(\mathrm{QC})$ of $\mathrm{Tyr}+7 \mathrm{H}_{2} \mathrm{O}$ (indigo) ${ }^{43}$ and Tyr in solution (magenta). 49 Other panels show out-of-plane vibrational modes from NMA for each FF set, labelled as $\left(K_{\mathrm{imp}},\left\{K_{\mathrm{tor}}-K_{\mathrm{tor}}^{C 36}\right\}\right)$ and averaged using MD of aqueous solution over $200 \mathrm{ps.} \mathrm{Similar} \mathrm{modes} \mathrm{are} \mathrm{shown} \mathrm{with} \mathrm{the}$ same letter. Not Available indicates ranges in which no vibrations were reported in the cited literature. 
(Movie TYR33, $883 \mathrm{~cm}^{-1}$ ), and O (Movie TYR34, $890 \mathrm{~cm}^{-1}$ ). The mode at $830 \mathrm{~cm}^{-1}$ in the RS panel $\left(850 \mathrm{~cm}^{-1}\right.$ in the QC panel) includes out-of-plane vibrations of atoms $\mathrm{CZ}$ and $\mathrm{OH}^{43}$ that appear in mode M (Movie TYR32) in the C36 panel; these largely involve $\mathrm{C}-\mathrm{O}$ and $\mathrm{C}-\mathrm{H}$ motions away from the ring plane. Modes I (Movie TYR27), J (Movie TYR28), and K (Movie TYR29) in the C36 panel did not exist in the RS and QC results of Hernández et al. ${ }^{[43}$ Instead, we use general descriptions for modes in p-tyrosine that are provided by Yao et al. $\frac{49}{4}$ (as a comparison within their study of m-tyrosine) to assign these modes to their corresponding RS, IR, and QC spectra. Mode I at $742 \mathrm{~cm}^{-1}$ in the RS panel $\left(739 \mathrm{~cm}^{-1}\right.$ at $741 \mathrm{~cm}^{-1}$ in the QC and IR panels) includes out-ofplane deformation of the ring. This mode in the C36 panels involves torsional distortions of the ring at the linker and $\mathrm{OH}$ group. Mode $\mathrm{K}$ at $832 \mathrm{~cm}^{-1}$ in the RS panel $\left(831 \mathrm{~cm}^{-1}\right.$ in each of the QC and IR panels) includes the breathing and out-of-plane ring vibrations of carbon and hydrogen atoms. 49 Mode $\mathrm{K}$ in the C36 panels has a slight breathing vibration and out-of-plane ring vibration. In modes $\mathrm{J}$ and $\mathrm{K}$, atoms in the ring of Tyr have very slight participation compared to backbone/linker vibrations. Hence, changes in $d_{\perp}$ were small in Fig. S11. ESI $\dagger$. Modes of Yao et al. that were not explained by Hernández et al. are represented by grey, magenta, and brown colors in RS, QC, and IR panels, respectively.

Mode H (Movie TYR27) in the RS, IR, QC, and C36 panels corresponds to out-of-plane vibrations of atoms in the aromatic ring and especially to CB-CG and CZ-OH out-of-plane bending. Modes A-G that were described by Yao et al., 49 yet were not presented by Hernández et al., 43 have corresponding modes using the C36 FF. Modes D-G are more related to out-of-plane deformation of the ring, with more deformation at $\mathrm{CZ}-\mathrm{OH}$ occurring in modes $\mathrm{F}$ and $\mathrm{G}$, while modes A-C are related to wagging of the whole ring. 49 Mode A has a very high standard deviation in all panels. Animation of this mode (Movie TYR09) shows an out-of-plane vibration around bond CB-CG. Finding similar modes during a 200 ps simulation only on the basis of the dot product is not sufficient for targeting this mode with precision.

A difference in the pattern and the frequencies for Tyr with the C36 FF compared to RS, IR, and QC can be observed in Fig. 16. Modes L-R appear at higher frequencies while other modes, such as H-K, appear at lower frequencies. The NMA results of NVE MD simulations for Tyr (Fig. S13, ESI $\dagger$ ) were consistent with NPT MD simulation results, confirming independence of out-of-plane ring atom vibrations from the temperature and pressure control algorithms. In addition, the NMA results on Tyr with methylamide terminal groups in solution (Fig. S13, ESI $\dagger$ ) show that many frequencies of out-of-plane vibrations are similar to those of the Tyr zwitterion for the C36 FF. Frequency patterns for both architectures differ by a similar extent from RS, IR, and QC results.

Applying an improper torsion potential disturbs the pattern of frequencies even more, and the modes were shifted generally to higher frequencies. This was similar to the Trp results: by increasing $K_{\text {imp }}$, out-of-plane displacements of atoms in the ring decreased and atoms remained closer to a planar structure, speeds of out-of-plane displacements increased, and as a consequence out-of-plane modes shifted to higher frequencies.

We applied changes in $K_{\text {tor }}$ to all except the smallest torsion angle potentials (Table 2 and related discussion). The results in Fig. 16 show that the FF set in which improper torsion potentials with $K_{\text {imp }}=10 \mathrm{kcal} / \mathrm{mol}_{-} \mathrm{rad}^{2}$ were included for atoms in the ring of Tyr, and $K_{\mathrm{tor}}$ of torsion angle sets around the aromatic ring were decreased by $1 \mathrm{kcal} / \mathrm{mol}$, provided the best match of pattern and out-of-plane frequencies compared with published RS and QC spectra. The results from geometric analysis illustrated that this FF set did not change planarity of the Tyr ring. Force field parameters that decrease out-of-plane fluctuations while achieving proper vibrational frequencies have not yet been demonstrated for Tyr.

The frequencies calculated by Fourier transformation of time autocorrelation functions for the out-of-plane displacement are in agreement with the results of Fig. 16 For example, out-of-plane vibrations of atoms $\mathrm{CB}, \mathrm{CG}, \mathrm{CD} 1$, and $\mathrm{CD} 2$ of Tyr are shown in Fig. 11 for different FF sets. Its C36 $(0,0)$ panel shows 4 sharp peaks around $475,508,665$, and $724 \mathrm{~cm}^{-1}$ that correspond to modes F, G, H, and J with similar frequencies in Fig.16. Applying FF set $(10,-1)$ shifted the peak at $508 \mathrm{~cm}^{-1}$ to a lower frequency (Fig. 11). The $(10,-1)$ panel of Fig. 16 confirms this small shift. Motions similar to those of mode $\mathrm{H}$ in FF $(0,0)$ appeared in three modes in $(10,-1)$ at different frequencies. One of these $\mathrm{H}$ modes shifted to a lower frequency, similar to Fig. 11 . The other two $\mathrm{H}$ modes did not shift in frequency. A similar interpretation can be applied to the results of Fig. S9 in ESI and Fig. 16.

\section{Conclusions}

Molecular dynamics (MD) simulations on the basis of the CHARMM36 Force Field (C36 FF) show that atoms in the heteroaromatic rings of tryptophan (Trp, W) and tyrosine (Tyr, Y) do not form planar structures. Here we show by using a geometric analysis that the largest deviations from planarity in these MD simulations occurred around the nitrogen atom (NE1) in the Trp ring and the gamma carbon (CG) in the Tyr ring.

C36 FF does not incorporate an improper torsion angle potential for atoms in the rings of the amino acids Trp and Tyr. We investigated effects of improper torsion angle potential on planarity by testing different improper force constants $\left(K_{\text {imp }}\right)$ in MD simulations. The average sums of angles between bonds around ring atoms in both Trp and Tyr molecules became closer to the planar limit of $360^{\circ}$, and the average displacements of these atoms from a planar structure decreased, after improper torsion terms were included. In summary, more planar structures for the rings of Trp and Tyr arise when improper torsion terms impose restoration forces, and rings become more planar as $K_{\text {imp }}$ increases. Results for the radial distribution function $g(r)$ between atoms in the rings and atoms in water molecules show that larger vs. smaller out-of-plane vibrations of atoms in the aromatic rings affect the concentration and proximity of nearby molecules and, consequently, impact interactions between atoms in the rings of Trp and Tyr and neighboring molecules.

In addition, time autocorrelation functions of the distance of each central atom from a plane containing its three connected atoms $\left(d_{\perp}\right)$ were obtained from the simulations. Fourier transforms of these $d_{\perp}$ time autocorrelation functions indicated that out-of-plane displacement vibrations of atoms in the aromatic 
rings shifted to higher frequencies after including improper torsion terms compared to results using the C36 FF. By applying higher $K_{\text {imp }}$, out-of-plane displacements of atoms in the heteroaromatic rings decreased; however, the speeds of these displacements simultaneously increased, and as a consequence the out-of-plane vibrations shifted to higher frequencies. Furthermore, we found that decreasing the torsion angle force constant $\left(K_{\text {tor }}\right)$ could shift out-of-plane displacements of atoms in the rings to lower frequencies. Our calculations show that both torsion and improper torsion force constants work together to control out-ofplane displacement of atoms in the rings of these two amino acids. The magnitudes of out-of-plane fluctuations were comparable for Trp and Tyr as zwitterions and with methylamide terminal groups.

Although imposing improper torsion forces led to a significant decrease in nonplanarity of heteroaromatic rings of amino acid side chains, the frequency shifts of out-of-plane displacements made it crucial to study the effects of each FF set on inherent vibrational frequencies of ring atoms. Thus an all-atom normal mode analysis (NMA) package was developed to quantify the characteristic vibrational modes of these two aromatic amino acids in MD simulations.

To investigate effects of water molecules on the vibrational modes of Trp and Tyr molecules, NMA was implemented on minimized structures of Trp and Tyr both in vacuum and in aqueous solution on the basis of the C36 FF. Results indicated that eliminating water molecules in the NMA calculation did not have a significant effect on the calculated frequencies of out-of-plane vibrational modes of the heteroaromatic rings. Modes in the aqueous system remained distributed around the same frequencies that were found for the vacuum system.

NMA next was applied to individual Trp and Tyr molecules within equilibrated MD simulations of aqueous solutions. The results of NMA for both amino acids showed that the pattern and the frequencies of out-of-plane motions obtained by using the C36 FF were different from prior Raman spectroscopy (RS), 43/44/46/49 infrared spectroscopy,, $46 / 49$ and quantum calculation (QC) 43/44/46/49 results, with some out-of-plane vibrational motions having higher frequencies.

The C36 FF has not incorporated an improper torsion potential for atoms in the rings of Trp and Tyr. We applied this potential with different improper force constants $\left(K_{\mathrm{imp}}\right)$ in MD simulations and NMA calculations. Although including improper torsion led to significant decreases in nonplanarity of aromatic rings, out-of-plane vibrations of atoms in the aromatic rings shifted to even higher frequencies compared to the RS, IR, and QC results reported in the literature.43/44/46/49 This makes sense because applying a higher $K_{\text {imp }}$ decreased out-of-plane displacements of atoms in the ring; however, speeds of these displacements increased and, as a consequence, out-of-plane normal modes shifted to higher frequencies. Our calculations demonstrated that out-of-plane vibrational motions of atoms in the ring are affected by both improper torsion and torsion force constants. For both molecules, we have shown that reducing $K_{\text {tor }}$ compared to those in C36 and including an appropriate $K_{\text {imp }}$ can improve agreement of vibrational modes with the RS and the QC results.

For Trp, a FF set in which improper torsion potentials with
$K_{\mathrm{imp}}=15 \mathrm{kcal} / \mathrm{mol}^{-\mathrm{rad}^{2}}$ were included for atoms in the ring, and $K_{\text {tor }}$ of torsion angle sets around the aromatic ring were decreased by $1 \mathrm{kcal} / \mathrm{mol}$, provided the best match of vibrational pattern and calculated out-of-plane frequencies in comparison to RS and QC results. This FF set reduces nonplanarity around the NE1 atom but does not reduce nonplanarity of other atoms in the ring. A FF set in which improper torsion potentials with $K_{\mathrm{imp}}=10 \mathrm{kcal} / \mathrm{mol}$ $\operatorname{rad}^{2}$ were included for atoms in the ring of Tyr, and $K_{\text {tor }}$ of all torsion angle sets around the aromatic ring were decreased by $1 \mathrm{kcal} / \mathrm{mol}$, provided the best match of vibrational pattern and out-of-plane frequencies with RS and QC results. This FF set does not reduce the nonplanarity that we have observed for the C36 FF. In conclusion, including improper torsion and reducing ring atom torsion angle parameters for Trp and Tyr molecules can reduce nonplanarity to some extent and can attain frequencies and frequency patterns for out-of-plane motions of atoms in aromatic rings within classical MD simulations that are similar to those from RS, IR, and QC. The extent of planarity influences proximity of nearest neighbor water molecules to heteroaromatic rings and potentially has biological consequences on the packing and folding of an active site.

\section{Conflicts of interest}

There are no conflicts to declare.

\section{Acknowledgements}

FJ acknowledges support from the URI Graduate School EGRA program. MLG acknowledges support from the US Fulbright Program.

\section{Notes and references}

1 X. Zhu, P. E. M. Lopes and A. D. MacKerell Jr., WIREs Comput. Mol. Sci., 2012, 2, 167-185.

2 O. Guvench and A. D. MacKerell Jr., in Molecular Modeling of Proteins, ed. A. Kokul, Humana Press, New York, 2008, pp. 63-88.

3 P. E. M. Lopes, O. Guvench and A. D. MacKerell Jr., Methods Mol. Biol., 2015, 1215, 47-71.

4 S. L. Mayo, B. D. Olafson and W. A. Goddard, J. Phys. Chem., 1990, 94, 8897-8909.

5 J. Li, R. Lakshminarayanan, Y. Bai, S. Liu, L. Zhou, K. Pervushin, C. Verma and R. W. Beuerman, J. Chem. Phys., 2012, 137, 215101.

6 A. D. MacKerell Jr., N. Banavali and N. Foloppe, Biopolymers, 2000, 56, 257-265.

7 R. B. Best, X. Zhu, J. Shim, P. E. M. Lopes, J. Mittal, M. Feig and A. D. MacKerell Jr., J. Chem. Theory Comput., 2012, 8, 3257-3273.

8 J. Huang and A. D. MacKerell Jr., J. Comput. Chem., 2013, 34, 2135-2145.

9 J. Huang, S. Rauscher, G. Nawrocki, T. Ran, M. Feig, B. L. de Groot, H. Grubmüller and A. D. MacKerell Jr., Nat. Methods, 2016, 14, 71-73.

10 E. J. Denning, U. D. Priyakumar, L. Nilsson and A. D. MacKerell Jr., J. Comput. Chem., 2011, 32, 1929-1943. 
11 K. Hart, N. Foloppe, C. M. Baker, E. J. Denning, L. Nilsson and A. D. Mackerell Jr., J. Chem. Theory Comput, 2012, 8, 348362.

12 Y. Wang, T. Zhao, D. Wei, E. Strandberg, A. S. Ulrich and J. P. Ulmschneider, Biochim. Biophys. Acta., 2014, 1838, 22802288.

13 A. D. MacKerell Jr., J. Comput. Chem., 2004, 25, 1584-1604.

14 R. B. Best, N.-V. Buchete and G. Hummer, Biophys. J., 2008, 95, L07-L09.

15 W. Chen, C. Shi, A. D. MacKerell Jr. and J. Shen, J. Phys. Chem. B, 2015, 119, 7902-7910.

16 S. Piana, K. Lindorff-Larsen and D. E. Shaw, Biophys. J., 2011, 100, L47-L49.

17 P. M. Hwang and H. J. Vogel, Biochem. Cell Biol., 1998, 76, 235-246.

18 P. L. Freddolino, S. Park, B. Roux and K. Schulten, Biophys. J., 2009, 96, 3772-3780.

19 X. Weng, L. Hamel, L. Martin and J. Peckham, J. Peckham, 2005 IEEE Congress on Evolutionary Computation, Edinburgh, UK, 2005, pp. 49-56.

20 K. Kachel, E. Asuncion-Punzalan and E. London, Biochemistry, 1995, 34, 15475-15479.

21 H. Hong, S. Park, R. H. Flores Jiménez, D. Rinehart and L. K. Tamm, J. Am. Chem. Soc., 2007, 129, 8320-8327.

22 A. Chattopadhyay, S. S. Rawat, D. V. Greathouse, D. A. Kelkar and R. E. Koeppe II, Biophys. J., 2008, 95, 166-175.

23 A. J. Johnston, Y. R. Zhang, S. Busch, L. C. Pardo, S. Imberti and S. E. McLain, J. Phys. Chem. B, 2015, 119, 5979-5987.

24 P. La Rocca, P. C. Biggin, D. P. Tieleman and M. S. P. Sansom, Biochim. Biophys. Acta, 1999, 1462, 185-200.

25 C.-W. Tsai, N.-Y. Hsu, C.-H. Wang, C.-Y. Lu, Y. Chang, H.-H. G. Tsai and R.-C. Ruaan, J. Mol. Biol., 2009, 392, 837-854.

26 Y. Wang, D. E. Schlamadinger, J. E. Kim and J. A. McCammon, Biochim. Biophys. Acta, 2012, 1818, 1402-1409.

27 A. T. Macias and A. D. MacKerell Jr., J. Comput. Chem., 2005, 26, 1452-1463.

28 H. M. Khan, C. Grauffel, R. Broer, A. D. MacKerell Jr., R. W. A. Havenith and N. Reuter, J. Chem. Theory Comput., 2016, 12, 5585-5595.

29 M. A. Anderson, B. Ogbay, R. Arimoto, W. Sha, O. G. Kisselev, D. P. Cistola and G. R. Marshall, J. Am. Chem. Soc., 2006, 128, 7531-7541.

30 G. P. Bean, J. Org. Chem., 1998, 63, 2497-2506.

31 A. R. Katritzky, M. Karelson, S. Sild, T. M. Krygowski and K. Jug, J. Org. Chem, 1998, 63, 5228-5231.

32 F. A. Cotton, Chemical Applications of Group Theory, John Wiley \& Sons, New York, 3rd edn, 1990.

33 Y. Harada and Y. Litaka, Acta Cryst., 1977, B33, 244-247.

34 M. Souhassou, C. Lecomte, R. H. Blessing, A. Aubry, M.-m. Rohmer, R. Wiest, M. Bénard and M. Marraud, Acta Cryst., 1991, B47, 253-266.

35 W. Caminati and S. Di Bernardo, J. Mol. Struct., 1990, 240, 253-262.

36 L. S. Slater and P. R. Callis, J. Phys. Chem., 1995, 99, 8572-
8581.

37 L. Serrano-Andrés and B. O. Roos, J. Am. Chem. Soc., 1996, 118, 185-195.

38 R. E. Koeppe II, H. Sun, P. C. A. Van der Wel, E. M. Scherer, P. Pulay and D. V. Greathouse, J. Am. Chem. Soc., 2003, 125, 12268-12276.

39 M. Faizan and S. Ahmad, J. Mol. Struct., 2018, 1171, 315322.

40 C. Bois, Acta Cryst., 1970, B26, 2086-2092.

41 H. F. Hameka and J. O. Jensen, J. Mol. Struct., 1995, 331, 203-214.

42 D. D. Li and M. L. Greenfield, Energy Fuels, 2011, 25, 36983705.

43 B. Hernández, F. Pflüger, A. Adenier, S. G. Kruglik and M. Ghomi, J. Phys. Chem. B, 2010, 114, 15319-15330.

44 C.-H. Chuang and Y.-T. Chen, J. Raman Spectrosc., 2009, 40, 150-156.

45 X. Cao and G. Fischer, J. Phys. Chem. A, 1999, 103, 999510003.

46 P. Leyton, J. Brunet, V. Silva, C. Paipa, M. V. Castillo and S. A. Brandán, Spectrochim. Acta Part A, 2012, 88, 162-170.

47 N. Maiti, S. Thomas, J. A. Jacob, R. Chadha, T. Mukherjee and S. Kapoor, J. Colloid Interface Sci., 2012, 380, 141-149.

48 C. D. Contreras, A. E. Ledesma, H. E. Lanús, J. Zinczuk and S. A. Brandán, Vib. Spectrosc., 2011, 57, 108-115.

49 G. Yao, J. Zhang and Q. Huang, Spectrochim. Acta Part A, 2015, 151, 111-123.

50 O. V. Shishkin, P. Dopieralski, I. V. Omelchenko, L. Gorb, Z. Latajka and J. Leszczynski, J. Phys. Chem. Lett., 2011, 2, 2881-2884.

51 B. A. Kelch, N. L. Salimi and D. A. Agard, Proc. Natl. Acad. Sci. USA, 2012, 109, 9414-9419.

52 B. R. Brooks, R. E. Bruccoleri, B. D. Olafson, D. J. States, S. Swaminathan and M. Karplus, J. Comput. Chem., 1983, 4, 187-217.

53 A. D. MacKerell Jr., D. Bashford, M. Bellott, R. L. Dunbrack Jr., J. D. Evanseck, M. J. Field, S. Fischer, J. Gao, H. Guo, S. Ha, D. Joseph-McCarthy, L. Kuchnir, K. Kuczera, F. T. K. Lau, C. Mattos, S. Michnick, T. Ngo, D. T. Nguyen, B. Prodhom, W. E. Reiher III, B. Roux, M. Schlenkrich, J. C. Smith, R. Stote, J. Straub, M. Watanabe, J. Wiórkiewicz-Kuczera, D. Yin and M. Karplus, J. Phys. Chem. B, 1998, 102, 35863616.

54 A. R. Leach, Molecular Modelling. Principles and Applications, Prentice Hall, Harlow, England, 2nd edn, 2001.

55 L. Skjaerven, S. M. Hollup and N. Reuter, J. Mol. Struct., 2009, 898, 42-48.

56 H. R. Horton, L. A. Moran, K. G. Scrimgeour, M. D. Perry and J. D. Rawn, Principles of Biochemistry, Pearson Prentice Hall, Upper Saddle River, NJ, 4th edn, 2006.

57 G. Lamoureux, E. Harder, I. V. Vorobyov, B. Roux and A. D. MacKerell Jr., Chem. Phys. Lett., 2006, 418, 245-249.

58 J. C. Phillips, R. Braun, W. Wang, J. Gumbart, E. Tajkhorshid, E. Villa, C. Chipot, R. D. Skeel, L. Kalé and K. Schulten, J. 
Comput. Chem., 2005, 26, 1781-1802.

59 S. J. Plimpton, R. Pollock and M. Stevens, Proc Eighth SIAM Conf. Parallel Process. Sci. Comput., 1997.

60 G. J. Martyna, D. J. Tobias and M. L. Klein, J. Chem. Phys., 1994, 101, 4177-4189.

61 S. E. Feller, Y. Zhang, R. W. Pastor and B. R. Brooks, J. Chem. Phys., 1995, 103, 4613-4621.

62 R. M. Stratt, Acc. Chem. Res., 1995, 28, 201.

63 E. Anderson, Z. Bai, C. Bishof, S. Blackford, J. Demmel, J. Dongarra, J. Du Croz, A. Greenbaum, S. Hammarling,
A. McKenney and D. Sorensen, LAPACK Users's Guide, Society for Industrial and Applied Mathematics, 3rd edn, 1999.

64 W. Humphrey, A. Dalke and K. Schulten, J. Molec. Graph., 1996, 14, 33-38.

65 W. L. DeLano, The PyMOL Molecular Graphics System, Version 1.8, Schrödinger LLC, 2015.

66 M. Masoori and M. L. Greenfield, Mol. Simul., 2017, 43, 1485-1495.

67 A. Lautie and A. Novak, J. Chem. Phys., 1972, 561, 24792481. 